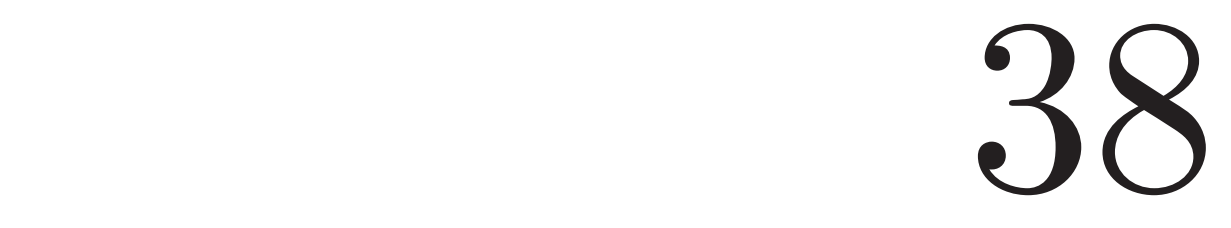

\title{
Cache-Oblivious Data Structures
}

Lars Arge

Duke University

Gerth Stølting Brodal

University of Aarhus

Rolf Fagerberg

University of Southern Denmark
38.1 The Cache-Oblivious Model ................... 38-1

38.2 Fundamental Primitives ......................... 38-3

Van Emde Boas Layout $\bullet k$-Merger

38.3 Dynamic B-Trees ............................... 38-8

Density Based $\bullet$ Exponential Tree Based

38.4 Priority Queues ............................. 38-12

Merge Based Priority Queue: Funnel Heap •

Exponential Level Based Priority Queue

38.5 2d Orthogonal Range Searching.................. 38-21

Cache-Oblivious kd-Tree • Cache-Oblivious Range Tree

\subsection{The Cache-Oblivious Model}

The memory system of most modern computers consists of a hierarchy of memory levels, with each level acting as a cache for the next; for a typical desktop computer the hierarchy consists of registers, level 1 cache, level 2 cache, level 3 cache, main memory, and disk. One of the essential characteristics of the hierarchy is that the memory levels get larger and slower the further they get from the processor, with the access time increasing most dramatically between main memory and disk. Another characteristics is that data is moved between levels in large blocks. As a consequence of this, the memory access pattern of an algorithm has a major influence on its practical running time. Unfortunately, the RAM model (Figure 38.1) traditionally used to design and analyze algorithms is not capable of capturing this, since it assumes that all memory accesses take equal time.

Because of the shortcomings of the RAM model, a number of more realistic models have been proposed in recent years. The most successful of these models is the simple two-level I/O-model introduced by Aggarwal and Vitter [2] (Figure 38.2). In this model the memory hierarchy is assumed to consist of a fast memory of size $M$ and a slower infinite memory, and data is transfered between the levels in blocks of $B$ consecutive elements. Computation

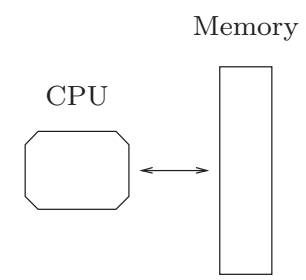

FIGURE 38.1: The RAM model.

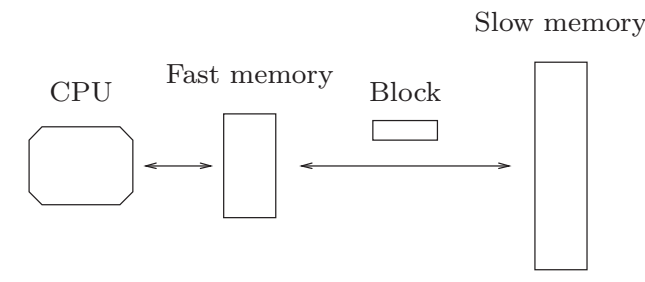

FIGURE 38.2: The I/O model. 
can only be performed on data in the fast memory, and it is assumed that algorithms have complete control over transfers of blocks between the two levels. We denote such a transfer a memory transfer. The complexity measure is the number of memory transfers needed to solve a problem. The strength of the $\mathrm{I} / \mathrm{O}$ model is that it captures part of the memory hierarchy, while being sufficiently simple to make design and analysis of algorithms feasible. In particular, it adequately models the situation where the memory transfers between two levels of the memory hierarchy dominates the running time, which is often the case when the size of the data exceeds the size of main memory. Agarwal and Vitter showed that comparison based sorting and searching require $\Theta\left(\operatorname{Sort}_{M, B}(N)\right)=\Theta\left(\frac{N}{B} \log _{M / B} \frac{N}{B}\right)$ and $\Theta\left(\log _{B} N\right)$ memory transfers in the I/O-model, respectively [2]. Subsequently a large number of other results have been obtained in the model; see the surveys by Arge [4] and Vitter [27] for references.

More elaborate models of multi-level memory than the I/O-model have been proposed (see e.g.[27] for an overview) but these models have been less successful, mainly because of their complexity. A major shortcoming of the proposed models, including the I/O-model, have also been that they assume that the characteristics of the memory hierarchy (the level and block sizes) are know. Very recently however, the cache-oblivious model, which assumes no knowledge about the hierarchy, was introduced by Frigo et al. [20]. In essence, a cache-oblivious algorithm is an algorithm formulated in the RAM model but analyzed in the I/O model, with the analysis required to hold for any $B$ and $M$. Memory transfers are assumed to be performed by an off-line optimal replacement strategy. The beauty of the cache-oblivious model is that since the I/O-model analysis holds for any block and memory size, it holds for all levels of a multi-level memory hierarchy (see [20] for details). In other words, by optimizing an algorithm to one unknown level of the memory hierarchy, it is optimized on all levels simultaneously. Thus the cache-oblivious model is effectively a way of modeling a complicated multi-level memory hierarchy using the simple two-level I/O-model.

Frigo et al. [20] described optimal $\Theta\left(\operatorname{Sort}_{M, B}(N)\right)$ memory transfer cache-oblivious algorithms for matrix transposition, fast Fourier transform, and sorting; Prokop also described a static search tree obtaining the optimal $O\left(\log _{B} N\right)$ transfer search bound [24]. Subsequently, Bender et al. [11] described a cache-oblivious dynamic search trees with the same search cost, and simpler and improved cache-oblivious dynamic search trees were then developed by several authors $[10,12,18,25]$. Cache-oblivious algorithms have also been developed for e.g. problems in computational geometry [1, 10, 15], for scanning dynamic sets [10], for layout of static trees [8], for partial persistence [10], and for a number of fundamental graph problems [5] using cache-oblivious priority queues [5, 16]. Most of these results make the so-called tall cache assumption, that is, they assume that $M>\Omega\left(B^{2}\right)$; we make the same assumption throughout this chapter.

Empirical investigations of the practical efficiency of cache-oblivious algorithms for sorting [19], searching [18, 23, 25] and matrix problems [20] have also been performed. The overall conclusion of these investigations is that cache-oblivious methods often outperform RAM algorithms, but not always as much as algorithms tuned to the specific memory hierarchy and problem size. On the other hand, cache-oblivious algorithms perform well on all levels of the memory hierarchy, and seem to be more robust to changing problem sizes than cache-aware algorithms.

In the rest of this chapter we describe some of the most fundamental and representative cache-oblivious data structure results. In Section 38.2 we discuss two fundamental primitives used to design cache-oblivious data structures. In Section 38.3 we describe two cache-oblivious dynamic search trees, and in Section 38.4 two priority queues. Finally, in Section 38.5 we discuss structures for 2-dimensional orthogonal range searching. 


\subsection{Fundamental Primitives}

The most fundamental cache-oblivious primitive is scanning-scanning an array with $N$ elements incurs $\Theta\left(\frac{N}{B}\right)$ memory transfers for any value of $B$. Thus algorithms such as median finding and data structures such as stacks and queues that only rely on scanning are automatically cache-oblivious. In fact, the examples above are optimal in the cacheoblivious model. Other examples of algorithms that only rely on scanning include Quicksort and Mergesort. However, they are not asymptotically optimal in the cache-oblivious model since they use $O\left(\frac{N}{B} \log \frac{N}{M}\right)$ memory transfers rather than $\Theta\left(\operatorname{Sort}_{M, B}(N)\right)$.

Apart from algorithms and data structures that only utilize scanning, most cache-oblivious results use recursion to obtain efficiency; in almost all cases, the sizes of the recursive problems decrease double-exponentially. In this section we describe two of the most fundamental such recursive schemes, namely the van Emde Boas layout and the $k$-merger.

\subsubsection{Van Emde Boas Layout}

One of the most fundamental data structures in the I/O-model is the B-tree [7]. A B-tree is basically a fanout $\Theta(B)$ tree with all leaves on the same level. Since it has height $O\left(\log _{B} N\right)$ and each node can be accessed in $O(1)$ memory transfers, it supports searches in $O\left(\log _{B} N\right)$ memory transfers. It also supports range queries, that is, the reporting of all $K$ elements in a given query range, in $O\left(\log _{B} N+\frac{K}{B}\right)$ memory transfers. Since $B$ is an integral part of the definition of the structure, it seems challenging to develop a cache-oblivious B-tree structure. However, Prokop [24] showed how a binary tree can be laid out in memory in order to obtain a (static) cache-oblivious version of a B-tree. The main idea is to use a recursively defined layout called the van Emde Boas layout closely related to the definition of a van Emde Boas tree [26]. The layout has been used as a basic building block of most cache-oblivious search structures (e.g in $[1,8,10,11,12,18,25])$.

\section{Layout}

For simplicity, we only consider complete binary trees. A binary tree is complete if it has $2^{h}-1$ nodes and height $h$ for some integer $h$. The basic idea in the van Emde Boas layout of a complete binary tree $\mathcal{T}$ with $N$ leaves is to divide $\mathcal{T}$ at the middle level and lay out the pieces recursively (Figure 38.3). More precisely, if $\mathcal{T}$ only has one node it is simply laid out as a single node in memory. Otherwise, $\operatorname{let} h=\log N$ be the height of $\mathcal{T}$. We define the top tree $\mathcal{T}_{0}$ to be the subtree consisting of the nodes in the topmost $\lfloor h / 2\rfloor$ levels of $\mathcal{T}$, and the bottom trees $\mathcal{T}_{1}, \ldots, \mathcal{T}_{k}$ to be the $\Theta(\sqrt{N})$ subtrees rooted in the nodes on level $\lceil h / 2\rceil$ of $\mathcal{T}$; note that all the subtrees have size $\Theta(\sqrt{N})$. The van Emde Boas layout of $\mathcal{T}$ consists of the van Emde Boas layout of $\mathcal{T}_{0}$ followed by the van Emde Boas layouts of $\mathcal{T}_{1}, \ldots, \mathcal{T}_{k}$.

\section{Search}

To analyze the number of memory transfers needed to perform a search in $\mathcal{T}$, that is, traverse a root-leaf path, we consider the first recursive level of the van Emde boas layout where the subtrees are smaller than $B$. As this level $\mathcal{T}$ is divided into a set of base trees of size between $\Theta(\sqrt{B})$ and $\Theta(B)$, that is, of height $\Omega(\log B)$ (Figure 38.4). By the definition of the layout, each base tree is stored in $O(B)$ contiguous memory locations and can thus be accessed in $O(1)$ memory transfers. That the search is performed in $O\left(\log _{B} N\right)$ memory transfers then follows since the search path traverses $O((\log N) / \log B)=O\left(\log _{B} N\right)$ different base trees. 
$38-4$

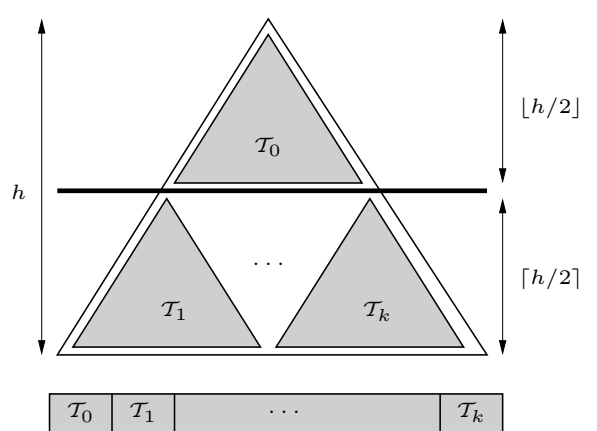

FIGURE 38.3: The van Emde Boas layout.

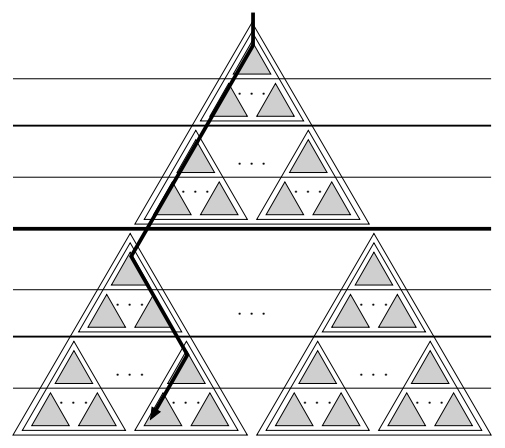

FIGURE 38.4: A search path.

\section{Range query}

To analyze the number of memory transfers needed to answer a range query $\left[x_{1}, x_{2}\right]$ on $\mathcal{T}$ using the standard recursive algorithm that traverses the relevant parts of $\mathcal{T}$ (starting at the root), we first note that the two paths to $x_{1}$ and $x_{2}$ are traversed in $O\left(\log _{B} N\right)$ memory transfers. Next we consider traversed nodes $v$ that is not on the two paths to $x_{1}$ and $x_{2}$. Since all elements in the subtree $\mathcal{T}_{v}$ rooted at such a node $v$ are reported, and since a subtree of height $\log B$ stores $\Theta(B)$ elements, $O\left(\frac{K}{B}\right)$ subtrees $\mathcal{T}_{v}$ of height $\log B$ are visited. This in turn means that the number of visited nodes above the last $\log B$ levels of $\mathcal{T}$ is also $O\left(\frac{K}{B}\right)$; thus they can all be accessed in $O\left(\frac{K}{B}\right)$ memory transfers. Consider the smallest recursive level of the van Emde Boas layout that completely contain $\mathcal{T}_{v}$. This level is of size between $\Omega(B)$ and $O\left(B^{2}\right)$ (Figure 38.5(a)). On the next level of recursion $\mathcal{T}_{v}$ is broken into a top part and $O(\sqrt{B})$ bottom parts of size between $\Omega(\sqrt{B})$ and $O(B)$ each (Figure 38.5(b)). The top part is contained in a recursive level of size $O(B)$ and is thus stored within $O(B)$ consecutive memory locations; therefore it can be accessed in $O(1)$ memory accesses. Similarly, the $O(B)$ nodes in the $O(\sqrt{B})$ bottom parts are stored consecutively in memory; therefore they can all be accessed in a total of $O(1)$ memory transfers. Therefore, the optimal paging strategy can ensure that any traversal of $\mathcal{T}_{v}$ is performed in $O(1)$ memory transfers, simply by accessing the relevant $O(1)$ blocks. Thus overall a range query is performed in $O\left(\log _{B} N+\frac{K}{B}\right)$ memory transfers.

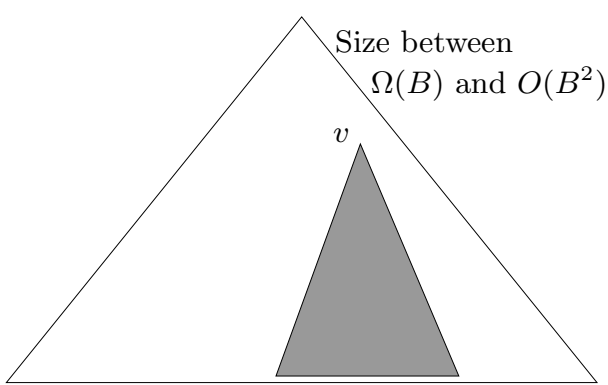

(a)

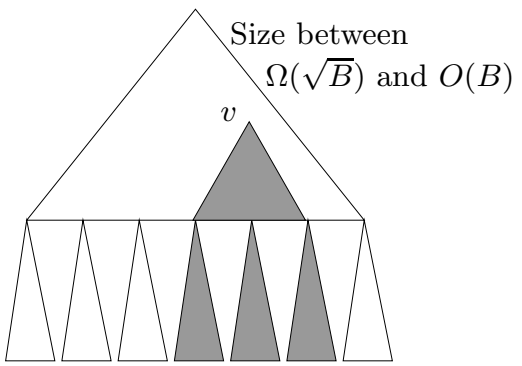

(b)

FIGURE 38.5: Traversing tree $\mathcal{T}_{v}$ with $O(B)$ leaves; (a) smallest recursive van Emde Boas level containing $\mathcal{T}_{v}$ has size between $\Omega(B)$ and $O\left(B^{2}\right)$; (b) next level in recursive subdivision. 


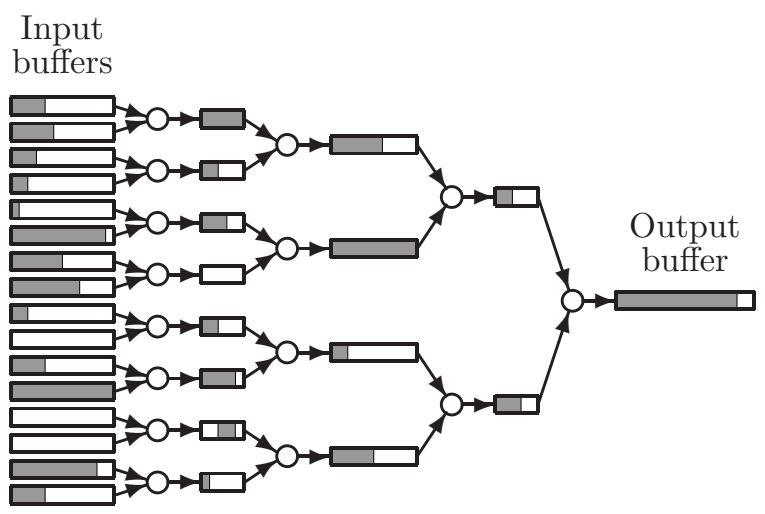

FIGURE 38.6: A 16-merger consisting of 15 binary mergers. Shaded parts represent elements in buffers.

THEOREM 38.1 Let $\mathcal{T}$ be a complete binary tree with $N$ leaves laid out using the van Emde Boas layout. The number of memory transfers needed to perform a search (traverse a root-to-leaf path) and a range query in $\mathcal{T}$ is $O\left(\log _{B} N\right)$ and $O\left(\log _{B} N+\frac{K}{B}\right)$, respectively.

The navigation from node to node in the van Emde Boas layout is straight-forward if the tree is implemented using pointers. However, navigation using arithmetic on array indexes is also possible [18]. This avoids the use of pointers and hence saves space.

The constant in the $O\left(\log _{B} N\right)$ bound for searching in Theorem 38.1 can be seen to be four. Further investigations of which constants are possible for cache-oblivious comparison based searching appear in [9].

\subsection{2 $k$-Merger}

In the I/O-model the two basic optimal sorting algorithms are multi-way versions of Mergesort and distribution sorting (Quicksort) [2]. Similarly, Frigo et al. [20] showed how both merge based and distribution based optimal cache-oblivious sorting algorithms can be developed. The merging based algorithm, Funnelsort, is based on a so-called $k$-merger. This structure has been used as a basic building block in several cache-oblivious algorithms. Here we describe a simplified version of the $k$-merger due to Brodal and Fagerberg [15].

\section{Binary mergers and merge trees}

A binary merger merges two sorted input streams into a sorted output stream: In one merge step an element is moved from the head of one of the input streams to the tail of the output stream; the heads of the input streams, as well as the tail of the output stream, reside in buffers of a limited capacity.

Binary mergers can be combined to form binary merge trees by letting the output buffer of one merger be the input buffer of another - in other words, a binary merge tree is a binary tree with mergers at the nodes and buffers at the edges, and it is used to merge a set of sorted input streams (at the leaves) into one sorted output stream (at the root). Refer to Figure 38.6 for an example.

An invocation of a binary merger in a binary merge tree is a recursive procedure that performs merge steps until the output buffer is full (or both input streams are exhausted); if 


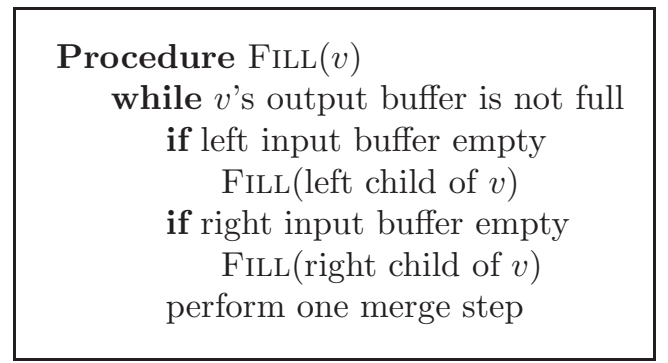

FIGURE 38.7: Invocation of binary merger $v$.

an input buffer becomes empty during the invocation (and the corresponding stream is not exhausted), the input buffer is recursively filled by an invocation of the merger having this buffer as output buffer. If both input streams of a merger get exhausted, the corresponding output stream is marked as exhausted. A procedure FilL $(v)$ performing an invocation of a binary merger $v$ is shown in Figure 38.7 (ignoring exhaustion issues). A single invocation FiLL $(r)$ on the root $r$ of a merge tree will merge the streams at the leaves of the tree.

\section{$k$-merger}

A $k$-merger is a binary merge tree with specific buffer sizes. For simplicity, we assume that $k$ is a power of two, in which case a $k$-merger is a complete binary tree of $k-1$ binary mergers. The output buffer at the root has size $k^{3}$, and the sizes of the rest of the buffers are defined recursively in a manner resembling the definition of the van Emde Boas layout: Let $i=\log k$ be the height of the $k$-merger. We define the top tree to be the subtree consisting of all mergers of depth at most $\lceil i / 2\rceil$, and the bottom trees to be the subtrees rooted in nodes at depth $\lceil i / 2\rceil+1$. We let the edges between the top and bottom trees have buffers of size $k^{3 / 2}$, and define the sizes of the remaining buffers by recursion on the top and bottom trees. The input buffers at the leaves hold the input streams and are not part of the $k$-merger definition. The space required by a $k$-merger, excluding the output buffer at the root, is given by $S(k)=k^{1 / 2} \cdot k^{3 / 2}+\left(k^{1 / 2}+1\right) \cdot S\left(k^{1 / 2}\right)$, which has the solution $S(k)=\Theta\left(k^{2}\right)$.

We now analyze the number of memory transfers needed to fill the output buffer of size $k^{3}$ at the root of a $k$-merger. In the recursive definition of the buffer sizes in the $k$-merger, consider the first level where the subtrees (excluding output buffers) have size less than $M / 3$; if $\bar{k}$ is the number of leaves of one such subtree, we by the space usage of $k$-mergers have $\bar{k}^{2} \leq M / 3$ and $\left(\bar{k}^{2}\right)^{2}=\bar{k}^{4}=\Omega(M)$. We call these subtrees of the $k$-merger base trees and the buffers between the base trees large buffers. Assuming $B^{2} \leq M / 3$, a base tree $\mathcal{T}_{v}$ rooted in $v$ together with one block from each of the large buffers surrounding it (i.e., its single output buffer and $\bar{k}$ input buffers) can be contained in fast memory, since $M / 3+B+\bar{k} \cdot B \leq M / 3+B+(M / 3)^{1 / 2} \cdot(M / 3)^{1 / 2} \leq M$.

If the $k$-merger consist of a single base tree, the number of memory transfers used to fill its output buffer with $k^{3}$ elements during an invocation is trivially $O\left(k^{3} / B+k\right)$. Otherwise, consider an invocation of the root $v$ of a base tree $\mathcal{T}_{v}$, which will fill up the size $\Omega\left(\bar{k}^{3}\right)$ output buffer of $v$. Loading $\mathcal{T}_{v}$ and one block for each of the $\bar{k}$ buffers just below it into fast memory will incur $O\left(\bar{k}^{2} / B+\bar{k}\right)$ memory transfers. This is $O(1 / B)$ memory transfer for each of the $\Omega\left(\bar{k}^{3}\right)$ elements output, since $\bar{k}^{4}=\Omega(M)$ implies $\bar{k}^{2}=\Omega\left(M^{1 / 2}\right)=\Omega(B)$, from which $\bar{k}=O\left(\bar{k}^{3} / B\right)$ follows. Provided that none of the input buffers just below $\mathcal{T}_{v}$ become 
empty, the output buffer can then be filled in $O\left(\bar{k}^{3} / B\right)$ memory transfers since elements can be read from the input buffers in $O(1 / B)$ transfers amortized. If a buffer below $\mathcal{T}_{v}$ becomes empty, a recursive invocation is needed. This invocation may evict $\mathcal{T}_{v}$ from memory, leading to its reloading when the invocation finishes. We charge this cost to the $\Omega\left(\bar{k}^{3}\right)$ elements in the filled buffer, or $O(1 / B)$ memory transfers per element. Finally, the last time an invocation is used to fill a particular buffer, the buffer may not be completely filled (due to exhaustion). However, this happens only once for each buffer, so we can pay the cost by charging $O(1 / B)$ memory transfers to each position in each buffer in the $k$-merger. As the entire $k$-merger uses $O\left(k^{2}\right)$ space and merges $k^{3}$ elements, these charges add up to $O(1 / B)$ memory transfers per element.

We charge an element $O(1 / B)$ memory transfers each time it is inserted into a large buffer. Since $\bar{k}=\Omega\left(M^{1 / 4}\right)$, each element is inserted in $O\left(\log _{\bar{k}} k\right)=O\left(\log _{M} k^{3}\right)$ large buffers. Thus we have the following.

THEOREM 38.2 Excluding the output buffers, the size of a $k$-merger is $O\left(k^{2}\right)$ and it performs $O\left(\frac{k^{3}}{B} \log _{M} k^{3}+k\right)$ memory transfers during an invocation to fill up its output buffer of size $k^{3}$.

\section{Funnelsort}

The cache-oblivious sorting algorithm Funnelsort is easily obtained once the $k$-merger structure is defined: Funnelsort breaks the $N$ input elements into $N^{1 / 3}$ groups of size $N^{2 / 3}$, sorts them recursively, and then merges the sorted groups using an $N^{1 / 3}$-merger.

Funnelsort can be analyzed as follows: Since the space usage of a $k$-merger is sub-linear in its output, the elements in a recursive sort of size $M / 3$ only needs to be loaded into memory once during the entire following recursive sort. For $k$-mergers at the remaining higher levels in the recursion tree, we have $k^{3} \geq M / 3 \geq B^{2}$, which implies $k^{2} \geq B^{4 / 3}>B$ and hence $k^{3} / B>k$. By Theorem 38.2, the number of memory transfers during a merge involving $N^{\prime}$ elements is then $O\left(\log _{M}\left(N^{\prime}\right) / B\right)$ per element. Hence, the total number of memory transfers per element is

$$
O\left(\frac{1}{B}\left(1+\sum_{i=0}^{\infty} \log _{M} N^{(2 / 3)^{i}}\right)\right)=O\left(\left(\log _{M} N\right) / B\right)
$$

Since $\log _{M} x=\Theta\left(\log _{M / B} x\right)$ when $B^{2} \leq M / 3$, we have the following theorem.

THEOREM 38.3 Funnelsort sorts $N$ element using $O\left(\operatorname{Sort}_{M, B}(N)\right)$ memory transfers.

In the above analysis, the exact (tall cache) assumption on the size of the fast memory is $B^{2} \leq M / 3$. In [15] it is shown how to generalize Funnelsort such that it works under the weaker assumption $B^{1+\varepsilon} \leq M$, for fixed $\varepsilon>0$. The resulting algorithm incurs the optimal $O\left(\operatorname{Sort}_{M, B}(N)\right)$ memory transfers when $B^{1+\varepsilon}=M$, at the price of incurring $O\left(\frac{1}{\varepsilon} \cdot \operatorname{Sort}_{M, B}(N)\right)$ memory transfers when $B^{2} \leq M$. It is shown in [17] that this trade-off is the best possible for comparison based cache-oblivious sorting. 


\subsection{Dynamic B-Trees}

The van Emde Boas layout of a binary tree provides a static cache-oblivious version of $B$-trees. The first dynamic solution was given Bender et al. [11], and later several simplified structures were developed $[10,12,18,25]$. In this section, we describe two of these structures $[10,18]$.

\subsubsection{Density Based}

In this section we describe the dynamic cache-oblivious search tree structure of Brodal et al [18]. A similar proposal was given independently by Bender et al. [12].

The basic idea in the structure is to embed a dynamic binary tree of height $\log N+O(1)$ into a static complete binary tree, that is, in a tree with $2^{h}-1$ nodes and height $h$, which in turn is embedded into an array using the van Emde Boas layout. Refer to Figure 38.8.

To maintain the dynamic tree we use techniques for maintaining small height in a binary tree developed by Andersson and Lai [3]; in a different setting, similar techniques has also been given by Itai et al. [21]. These techniques give an algorithm for maintaining height $\log N+O(1)$ using amortized $O\left(\log ^{2} N\right)$ time per update. If the height bound is violated after performing an update in a leaf $l$, this algorithm performs rebalancing by rebuilding the subtree rooted at a specific node $v$ on the search path from the root to $l$. The subtree is rebuilt to perfect balance in time linear in the size of the subtree. In a binary tree of perfect balance the element in any node $v$ is the median of all the elements stored in the subtree $\mathcal{T}_{v}$ rooted in $v$. This implies that only the lowest level in $\mathcal{T}_{v}$ is not completely filled and the empty positions appearing at this level are evenly distributed across the level. Hence, the net effect of the rebuilding is to redistribute the empty positions in $\mathcal{T}_{v}$. Note that this can lower the cost of future insertions in $\mathcal{T}_{v}$, and consequently it may in the long run be better to rebuild a subtree larger than strictly necessary for reestablishment of the height bound. The criterion for choosing how large a subtree to rebuild, i.e. for choosing the node $v$, is the crucial part of the algorithms by Andersson and Lai [3] and Itai et al. [21]. Below we give the details of how they can be used in the cache-oblivious setting.

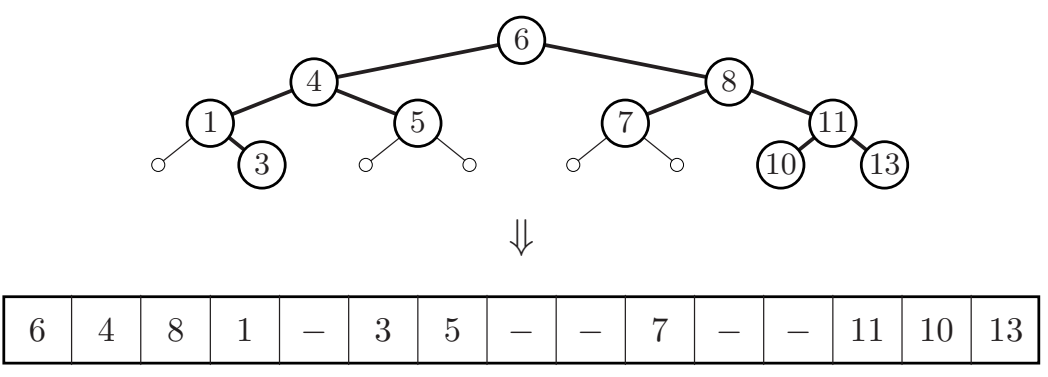

FIGURE 38.8: Illustration of embedding a height $H$ tree into a complete static tree of height $H$, and the van Emde Boas layout of this tree.

\section{Structure}

As mentioned, our data structure consists of a dynamic binary tree $\mathcal{T}$ embedded into a static complete binary tree $\mathcal{T}^{\prime}$ of height $H$, which in turn is embedded into an array using the van Emde Boas layout. 
In order to present the update and query algorithms, we define the density $\rho(u)$ of a node $u$ as $\left|T_{u}\right| /\left|T_{u}^{\prime}\right|$, where $\left|T_{u}\right|$ and $\left|T_{u}^{\prime}\right|$ are the number of nodes in the trees rooted in $u$ in $\mathcal{T}$ and $\mathcal{T}^{\prime}$, respectively. In Figure 38.8, the node containing the element 4 has balance $4 / 7$. We also define two density thresholds $\tau_{i}$ and $\gamma_{i}$ for the nodes on each level $i=1,2, \ldots, H$ (where the root is at level 1 ). The upper density thresholds $\tau_{i}$ are evenly space values between $3 / 4$ and 1 , and the lower density thresholds $\gamma_{i}$ are evenly spaced values between $1 / 4$ and $1 / 8$. More precisely, $\tau_{i}=3 / 4+(i-1) /(4(H-1))$ and $\gamma_{i}=1 / 4-(i-1) /(8(H-1))$.

\section{Updates}

To insert a new element into the structure we first locate the position in $\mathcal{T}$ of the new node $w$. If the insertion of $w$ violates the height bound $H$, we rebalance $\mathcal{T}$ as follows: First we find the lowest ancestor $v$ of $w$ satisfying $\gamma_{i} \leq \rho(v) \leq \tau_{i}$, where $i$ is the level of $v$. If no ancestor $v$ satisfies the requirement, we rebuild the entire structure, that is, $\mathcal{T}, \mathcal{T}^{\prime}$ and the layout of $\mathcal{T}^{\prime}$ : For $k$ the integer such that $2^{k} \leq N<2^{k+1}$ we choose the new height $H$ of the tree $\mathcal{T}^{\prime}$ as $k+1$ if $N \leq 5 / 4 \cdot 2^{k}$; otherwise we choose $H=k+2$. On the other hand, if the ancestor $v$ exists we rebuild $\mathcal{T}_{v}$ : We first create a sorted list of all elements in $\mathcal{T}_{v}$ by an in-order traversal of $\mathcal{T}_{v}$. The $\left\lceil\left|T_{v}\right| / 2\right\rceil$ th element becomes the element stored at $v$, the smallest $\left\lfloor\left(\left|T_{v}\right|-1\right) / 2\right\rfloor$ elements are recursively distributed in the left subtree of $v$, and the largest $\left\lceil\left(\left|T_{v}\right|-1\right) / 2\right\rceil$ elements are recursively distributed in the right subtree of $v$.

We can delete an element from the structure in a similar way: We first locate the node $w$ in $\mathcal{T}$ containing the element $e$ to be deleted. If $w$ is not a leaf and has a right subtree, we then locate the node $w^{\prime}$ containing the immediate successor of $e$ (the node reached by following left children in the right subtree of $w$ ), swap the elements in $w$ and $w^{\prime}$, and let $w=w^{\prime}$. We repeat this until $w$ is a leaf. If on the other hand $w$ is not a leaf but only has a left subtree, we instead repeatedly swap $w$ with the node containing the predecessor of $e$. Finally, we delete the leaf $w$ from $\mathcal{T}$, and rebalance the tree by rebuilding the subtree rooted at the lowest ancestor $v$ of $w$ satisfying satisfying $\gamma_{i} \leq \rho(v) \leq \tau_{i}$, where $i$ is the level of $v$; if no such node exists we rebuild the entire structure completely.

Similar to the proof of Andersson and Lai [3] and Itai et al. [21] that updates are performed in $O\left(\log ^{2} N\right)$ time, Brodal et al. [18] showed that using the above algorithms, updates can be performed in amortized $O\left(\log _{B} N+\left(\log ^{2} N\right) / B\right)$ memory transfers.

\section{Range queries}

In Section 38.2, we discussed how a range query can be answered in $O\left(\log _{B} N+\frac{K}{B}\right)$ memory transfers on a complete tree $\mathcal{T}^{\prime}$ laid out using the van Emde Boas layout. Since it can be shown that the above update algorithm maintains a lower density threshold of $1 / 8$ for all nodes, we can also perform range queries in $\mathcal{T}$ efficiently: To answer a range query $\left[x_{1}, x_{2}\right]$ we traverse the two paths to $x_{1}$ and $x_{2}$ in $\mathcal{T}$, as well as $O(\log N)$ subtrees rooted in children of nodes on these paths. Traversing one subtree $\mathcal{T}_{v}$ in $\mathcal{T}$ incurs at most the number of memory transfers needed to traverse the corresponding (full) subtree $\mathcal{T}_{v}^{\prime}$ in $\mathcal{T}^{\prime}$. By the lower density threshold of $1 / 8$ we know that the size of $\mathcal{T}_{v}^{\prime}$ is at most a factor of eight larger than the size of $\mathcal{T}_{v}$. Thus a range query is answered in $O\left(\log _{B} N+\frac{K}{B}\right)$ memory transfers.

THEOREM 38.4 There exists a linear size cache-oblivious data structure for storing $N$ elements, such that updates can be performed in amortized $O\left(\log _{B} N+\left(\log ^{2} N\right) / B\right)$ memory transfers and range queries in $O\left(\log _{B} N+\frac{K}{B}\right)$ memory transfers.

Using the method for moving between nodes in a van Emde Boas layout using arithmetic on the node indices rather than pointers, the data structure can be implemented as a 
single size $O(N)$ array of data elements. The amortized complexity of updates can also be lowered to $O\left(\log _{B} N\right)$ by changing leaves into pointers to buckets containing $\Theta(\log N)$ elements each. With this modification a search can still be performed in $O\left(\log _{B} N\right)$ memory transfers. However, then range queries cannot be answered efficiently, since the $O\left(\frac{K}{\log N}\right)$ buckets can reside in arbitrary positions in memory.

\subsubsection{Exponential Tree Based}

The second dynamic cache-oblivious search tree we consider is based on the so-called exponential layout of Bender et al. [10]. For simplicity, we here describe the structure slightly differently than in [10].

\section{Structure}

Consider a complete balanced binary tree $\mathcal{T}$ with $N$ leaves. Intuitively, the idea in an exponential layout of $\mathcal{T}$ is to recursively decompose $\mathcal{T}$ into a set of components, which are each laid out using the van Emde Boas layout. More precisely, we define component $\mathcal{C}_{0}$ to consist of the first $\frac{1}{2} \log N$ levels of $\mathcal{T}$. The component $\mathcal{C}_{0}$ contains $\sqrt{N}$ nodes and is called an $N$-component because its root is the root of a tree with $N$ leaves (that is, $\mathcal{T}$ ). To obtain the exponential layout of $\mathcal{T}$, we first store $\mathcal{C}_{0}$ using the van Emde Boas layout, followed immediately by the recursive layout of the $\sqrt{N}$ subtrees, $\mathcal{T}_{1}, \mathcal{I}_{2}, \ldots, \mathcal{T}_{\sqrt{N}}$, of size $\sqrt{N}$, beneath $\mathcal{C}_{0}$ in $\mathcal{T}$, ordered from left to right. Note how the definition of the exponential layout naturally defines a decomposition of $\mathcal{T}$ into $\log \log N+O(1)$ layers, with layer $i$ consisting of a number of $N^{1 / 2^{i-1}}$-components. An $X$-component is of size $\Theta(\sqrt{X})$ and its $\Theta(\sqrt{X})$ leaves are connected to $\sqrt{X}$-components. Thus the root of an $X$-component is the root of a tree containing $X$ elements. Refer to Figure 38.9. Since the described layout of $\mathcal{T}$ is really identical to the van Emde Boas layout, it follows immediately that it uses linear space and that a root-to-leaf path can be traversed in $O\left(\log _{B} N\right)$ memory transfers.

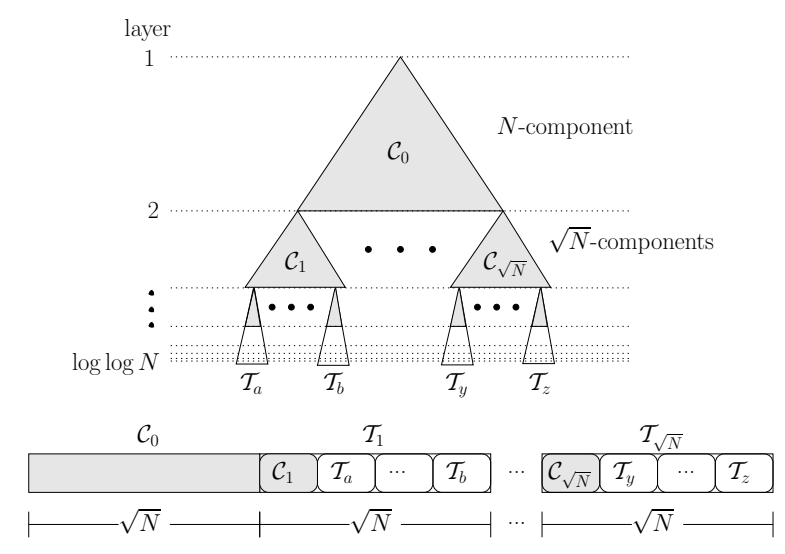

FIGURE 38.9: Components and exponential layout.

By slightly relaxing the requirements on the layout described above, we are able to maintain it dynamically: We define an exponential layout of a balanced binary tree $\mathcal{T}$ with $N$ leaves to consist of a composition of $\mathcal{T}$ into $\log \log N+O(1)$ layers, with layer $i$ consisting 
of a number of $N^{1 / 2^{i-1}}$-components, each laid out using the van Emde Boas layout (Figure 38.9). An $X$-component has size $\Theta(\sqrt{X})$ but unlike above we allow its root to be root in a tree containing between $X$ and $2 X$ elements. Note how this means that an $X$-component has between $X / 2 \sqrt{X}=\frac{1}{2} \sqrt{X}$ and $2 X / \sqrt{X}=2 \sqrt{X}$ leaves. We store the layout of $\mathcal{T}$ in memory almost as previously: If the root of $\mathcal{T}$ is root in an $X$-component $\mathcal{C}_{0}$, we store $\mathcal{C}_{0}$ first in $2 \cdot 2 \sqrt{X}-1$ memory locations (the maximal size of an $X$-component), followed immediately by the layouts of the subtrees $\left(\sqrt{X}\right.$-components) rooted in the leaves of $\mathcal{C}_{0}$ (in no particular order). We make room in the layout for the at most $2 \sqrt{X}$ such subtrees. This exponential layout for $\mathcal{T}$ uses $S(N)=\Theta(\sqrt{N})+2 \sqrt{N} \cdot S(\sqrt{N})$ space, which is $\Theta(N \log N)$.

\section{Search}

Even with the modified definition of the exponential layout, we can traverse any rootto-leaf path in $\mathcal{T}$ in $O\left(\log _{B} N\right)$ memory transfers: The path passes through exactly one $N^{1 / 2^{i-1}}$-component for $1 \leq i \leq \log \log N+O(1)$. Each $X$-component is stored in a van Emde Boas layout of size $\Theta(\sqrt{X})$ and can therefore be traversed in $\Theta\left(\log _{B} \sqrt{X}\right)$ memory transfers (Theorem 38.1). Thus, if we use at least one memory transfer in each component, we perform a search in $O\left(\log _{B} N\right)+\log \log N$ memory accesses. However, we do not actually use a memory transfer for each of the $\log \log N+O(1)$ components: Consider the traversed $X$-component with $\sqrt{B} \leq X \leq B$. This component is of size $O(\sqrt{B})$ and can therefore be loaded in $O(1)$ memory transfers. All smaller traversed components are of total size $O(\sqrt{B} \log \sqrt{B})=O(B)$, and since they are stored in consecutively memory locations they can also be traversed in $O(1)$ memory transfers. Therefore only $O(1)$ memory transfers are used to traverse the last $\log \log B-O(1)$ components. Thus, the total cost of traversing a root-to-leaf path is $O\left(\log _{B} N+\log \log N-\log \log B\right)=O\left(\log _{B} N\right)$.

\section{Updates}

To perform an insertion in $\mathcal{T}$ we first search for the leaf $l$ where we want to perform the insertion; inserting the new element below $l$ will increase the number of elements stored below each of the $\log \log N+O(1)$ components on the path to the root, and may thus result in several components needing rebalancing (an $X$-component with $2 X$ elements stored below it). We perform the insertion and rebalance the tree in a simple way as follows: We find the topmost $X$-component $\mathcal{C}_{j}$ on the path to the root with $2 X$ elements below it. Then we divide these elements into two groups of $X$ elements and store them separately in the exponential layout (effectively we split $\mathcal{C}_{j}$ with $2 X$ elements below it into two $X$-components with $X$ elements each). This can easily be done in $O(X)$ memory transfers. Finally, we update a leaf and insert a new leaf in the $X^{2}$-component above $\mathcal{C}_{j}$ (corresponding to the two new $X$-components); we can easily do so in $O(X)$ memory transfers by rebuilding it. Thus overall we have performed the insertion and rebalancing in $O(X)$ memory transfers. The rebuilding guarantees that after rebuilding an $X$-component, $X$ inserts have to be performed below it before it needs rebalancing again. Therefore we can charge the $O(X)$ cost to the $X$ insertions that occurred below $\mathcal{C}_{j}$ since it was last rebuilt, and argue that each insertion is charged $O(1)$ memory accesses on each of the $\log \log N+O(1)$ levels. In fact, using the same argument as above for the searching cost, we can argue that we only need to charge an insertion $O(1)$ transfers on the last $\log \log B-O(1)$ levels of $\mathcal{T}$, since rebalancing on any of these levels can always be performed in $O(1)$ memory transfers. Thus overall we perform an insertion in $O\left(\log _{B} N\right)$ memory transfers amortized.

Deletions can easily be handled in $O\left(\log _{B} N\right)$ memory transfers using global rebuilding: To delete the element in a leaf $l$ of $\mathcal{T}$ we simply mark $l$ as deleted. If $l$ 's sibling is also marked as deleted, we mark their parent deleted too; we continue this process along one path to the 
root of $\mathcal{T}$. This way we can still perform searches in $O\left(\log _{B} N\right)$ memory transfers, as long as we have only deleted a fraction of the elements in the tree. After $\frac{N}{2}$ deletes we therefore rebuild the entire structure in $O\left(N \log _{B} N\right)$ memory accesses, or $O\left(\log _{B} N\right)$ accesses per delete operation.

Bender et al. [10] showed how to modify the update algorithms to perform updates "lazily" and obtain worst case $O\left(\log _{B} N\right)$ bounds.

\section{Reducing space usage}

To reduce the space of the layout of a tree $\mathcal{T}$ to linear we simply make room for $2 \log N$ elements in each leaf, and maintain that a leaf contains between $\log N$ and $2 \log N$ elements. This does not increase the $O\left(\log _{B} N\right)$ search and update costs since the $O(\log N)$ elements in a leaf can be scanned in $O((\log N) / B)=O\left(\log _{B} N\right)$ memory accesses. However, it reduces the number of elements stored in the exponential layout to $O(N / \log N)$.

THEOREM 38.5 The exponential layout of a search tree $\mathcal{T}$ on $N$ elements uses linear space and supports updates in $O\left(\log _{B} N\right)$ memory accesses and searches in $O\left(\log _{B} N\right)$ memory accesses.

Note that the analogue of Theorem 38.1 does not hold for the exponential layout, i.e. it does not support efficient range queries. The reason is partly that the $\sqrt{X}$-components below an $X$-component are not located in (sorted) order in memory because components are rebalanced by splitting, and partly because of the leaves containing $\Theta(\log N)$ elements. However, Bender et al [10] showed how the exponential layout can be used to obtain a number of other important results: The structure as described above can easily be extended such that if two subsequent searched are separated by $d$ elements, then the second search can be performed in $O\left(\log ^{*} d+\log _{B} d\right)$ memory transfers. It can also be extended such that $R$ queries (batched searching) can be answered simultaneously in $O\left(R \log _{B} \frac{N}{R}+\operatorname{Sort}_{M, B}(R)\right)$ memory transfers. The exponential layout can also be used to develop a persistent $B$-tree, where updates can be performed in the current version of the structure and queries can be performed in the current as well as all previous versions, with both operations incurring $O\left(\log _{B} N\right)$ memory transfers. It can also be used as a basic building block in a linear space planar point location structure that answers queries in $O\left(\log _{B} N\right)$ memory transfers.

\subsection{Priority Queues}

A priority queue maintains a set of elements with a priority (or key) each under the operations InsERT and DeleteMin, where an InsERT operation inserts a new element in the queue, and a DELETEMIN operation finds and deletes the element with the minimum key in the queue. Frequently we also consider a DELETE operation, which deletes an element with a given key from the priority queue. This operation can easily be supported using INSERT and DeleteMin: To perform a Delete we insert a special delete-element in the queue with the relevant key, such that we can detect if an element returned by a DELETEMin has really been deleted by performing another DELETEMin.

A balanced search tree can be used to implement a priority queue. Thus the existence of a dynamic cache-oblivious B-tree immediately implies the existence of a cache-oblivious priority queue where all operations can be performed in $O\left(\log _{B} N\right)$ memory transfers, where $N$ is the total number of elements inserted. However, it turns out that one can design a priority queue where all operations can be performed in $\Theta\left(\operatorname{Sort}_{M, B}(N) / N\right)=O\left(\frac{1}{B} \log _{M / B} \frac{N}{B}\right)$ 
memory transfers; for most realistic values of $N, M$, and $B$, this bound is less than 1 and we can, therefore, only obtain it in an amortized sense. In this section we describe two different structures that obtain these bounds $[5,16]$.

\subsubsection{Merge Based Priority Queue: Funnel Heap}

The cache-oblivious priority queue Funnel Heap due to Brodal and Fagerberg [16] is inspired by the sorting algorithm Funnelsort [15, 20]. The structure only uses binary merging; essentially it is a heap-ordered binary tree with mergers in the nodes and buffers on the edges.

Link $i$

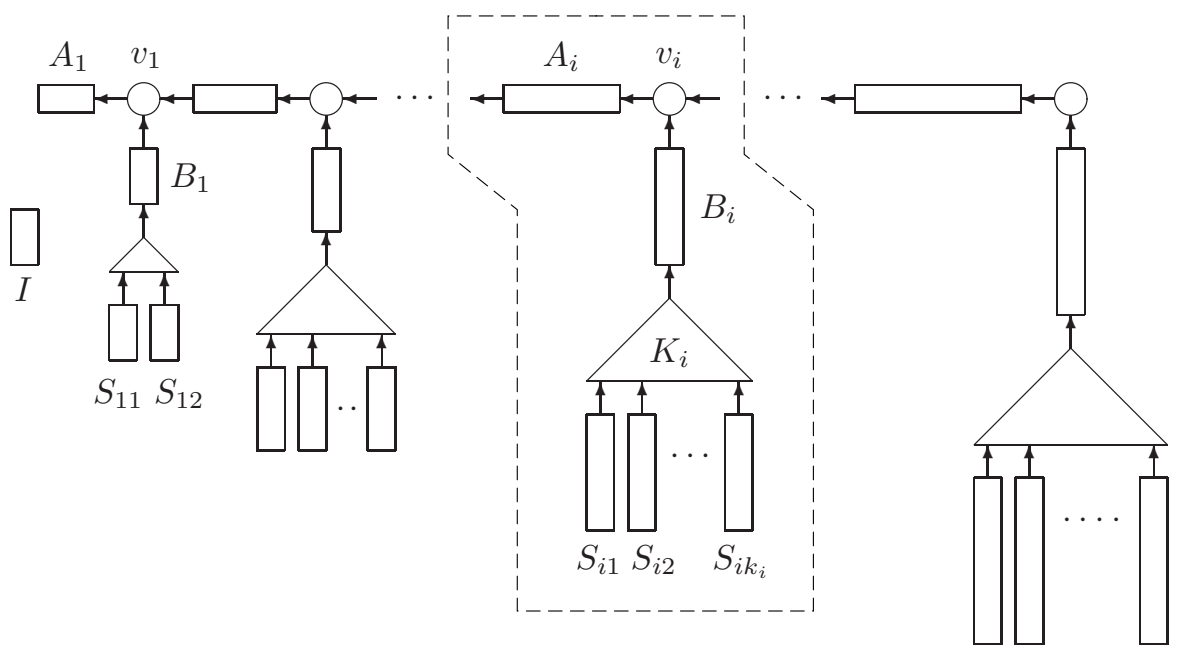

FIGURE 38.10: Funnel Heap: Sequence of $k$-mergers (triangles) linked together using buffers (rectangles) and binary mergers (circles).

\section{Structure}

The main part of the Funnel Heap structure is a sequence of $k$-mergers (Section 38.2.2) with double-exponentially increasing $k$, linked together in a list using binary mergers; refer to Figure 38.10. This part of the structure constitutes a single binary merge tree. Additionally, there is a single insertion buffer $I$.

More precisely, let $k_{i}$ and $s_{i}$ be values defined inductively by

$$
\begin{aligned}
\left(k_{1}, s_{1}\right) & =(2,8), \\
s_{i+1} & =s_{i}\left(k_{i}+1\right), \\
k_{i+1} & =\left\lceil\left\lceil s_{i+1} 1 / 3\right\rceil\right\rceil,
\end{aligned}
$$

where $\lceil x\rceil\rceil$ denotes the smallest power of two above $x$, i.e. $\left\lceil\lceil x\rceil=2^{\lceil\log x\rceil}\right.$. We note that $s_{i}{ }^{1 / 3} \leq k_{i}<2 s_{i}{ }^{1 / 3}$, from which $s_{i}{ }^{4 / 3}<s_{i+1}<3 s_{i}{ }^{4 / 3}$ follows, so both $s_{i}$ and $k_{i}$ grows double-exponentially: $s_{i+1}=\Theta\left(s_{i}^{4 / 3}\right)$ and $k_{i+1}=\Theta\left(k_{i}^{4 / 3}\right)$. We also note that by induction on $i$ we have $s_{i}=s_{1}+\sum_{j=1}^{i-1} k_{j} s_{j}$ for all $i$. 
A Funnel Heap consists of a linked list with link $i$ containing a binary merger $v_{i}$, two buffers $A_{i}$ and $B_{i}$, and a $k_{i}$-merger $K_{i}$ having $k_{i}$ input buffers $S_{i 1}, \ldots, S_{i k_{i}}$. We refer to $B_{i}$, $K_{i}$, and $S_{i 1}, \ldots, S_{i k_{i}}$ as the lower part of the link. The size of both $A_{i}$ and $B_{i}$ is $k_{i}^{3}$, and the size of each $S_{i j}$ is $s_{i}$. Link $i$ has an associated counter $c_{i}$ for which $1 \leq c_{i} \leq k_{i}+1$. The initial value of $c_{i}$ is one for all $i$. The structure also has one insertion buffer $I$ of size $s_{1}$. We maintain the following invariants:

Invariant 1 For link $i, S_{i c_{i}}, \ldots, S_{i k_{i}}$ are empty.

Invariant 2 On any path in the merge tree from some buffer to the root buffer $A_{1}$, elements appear in decreasing order.

Invariant 3 Elements in buffer I appear in sorted order.

Invariant 2 can be rephrased as the entire merge tree being in heap order. It implies that in all buffers in the merge tree, the elements appear in sorted order, and that the minimum element in the queue will be in $A_{1}$ or $I$, if buffer $A_{1}$ is non-empty. Note that an invocation (Figure 38.7 ) of any binary merger in the tree maintains the invariants.

\section{Layout}

The Funnel Heap is laid out in consecutive memory locations in the order $I$, link 1, link $2, \ldots$, with link $i$ being laid out in the order $c_{i}, A_{i}, v_{i}, B_{i}, K_{i}, S_{i 1}, S_{i 2}, \ldots, S_{i k_{i}}$.

\section{Operations}

To perform a DeLETEMin operation we compare the smallest element in $I$ with the smallest element in $A_{1}$ and remove the smallest of these; if $A_{1}$ is empty we first perform an invocation of $v_{1}$. The correctness of this procedure follows immediately from Invariant 2.

To perform an INSERT operation we insert the new element among the (constant number of) elements in $I$, maintaining Invariant 3. If the number of elements in $I$ is now $s_{1}$, we examine the links in order to find the lowest index $i$ for which $c_{i} \leq k_{i}$. Then we perform the following $\operatorname{SweEP}(i)$ operation.

In $\operatorname{Sweep}(i)$, we first traverse the path $p$ from $A_{1}$ to $S_{i c_{i}}$ and record how many elements are contained in each encountered buffer. Then we traverse the part of $p$ going from $A_{i}$ to $S_{i c_{i}}$, remove the elements in the encountered buffers, and form a sorted stream $\sigma_{1}$ of the removed elements. Next we form another sorted stream $\sigma_{2}$ of all elements in links $1, \ldots, i-1$ and in buffer $I$; we do so by marking $A_{i}$ temporarily as exhausted and calling DeLETEMin repeatedly. We then merge $\sigma_{1}$ and $\sigma_{2}$ into a single stream $\sigma$, and traverse $p$ again while inserting the front (smallest) elements of $\sigma$ in the buffers on $p$ such that they contain the same numbers of elements as before we emptied them. Finally, we insert the remaining elements from $\sigma$ into $S_{i c_{i}}$, reset $c_{l}$ to one for $l=1,2, \ldots, i-1$, and increment $c_{i}$.

To see that $\operatorname{Sweep}(i)$ does not insert more than the allowed $s_{i}$ elements into $S_{i c_{i}}$, first note that the lower part of link $i$ is emptied each time $c_{i}$ is reset to one. This implies that the lower part of link $i$ never contains more than the number of elements inserted into $S_{i 1}, S_{i 2}, \ldots, S_{i k_{i}}$ by the at most $k_{i} \operatorname{SweEP}(i)$ operations occurring since last time $c_{i}$ was reset. Since $s_{i}=s_{1}+\sum_{j=1}^{i-1} k_{j} s_{j}$ for all $i$, it follows by induction on time that no instance of $\operatorname{SweEP}(i)$ inserts more than $s_{i}$ elements into $S_{i c_{i}}$.

Clearly, $\operatorname{Sweep}(i)$ maintains Invariants 1 and 3, since $I$ and the lower parts of links $1, \ldots, i-1$ are empty afterwards. Invariant 2 is also maintained, since the new elements in the buffers on $p$ are the smallest elements in $\sigma$, distributed such that each buffer contains exactly the same number of elements as before the $\operatorname{SwEeP}(i)$ operation. After the operation, an element on this path can only be smaller than the element occupying the same location before the operation, and therefore the merge tree is in heap order. 


\section{Analysis}

To analyze the amortized cost of an INSERT or DELETEMIN operation, we first consider the number of memory transfers used to move elements upwards (towards $A_{1}$ ) by invocations of binary mergers in the merge tree. For now we assume that all invocations result in full buffers, i.e., that no exhaustions occur. We imagine charging the cost of filling a particular buffer evenly to the elements being brought into the buffer, and will show that this way an element from an input buffer of $K_{i}$ is charged $O\left(\frac{1}{B} \log _{M / B} s_{i}\right)$ memory transfers during its ascent to $A_{1}$.

Our proof of this will rely on the optimal replacement strategy keeping as many as possible of the first links of the Funnel Heap in fast memory at all times. To analyze the number of links that fit in fast memory, we define $\Delta_{i}$ to be the sum of the space used by links 1 to $i$ and define $i_{M}$ to be the largest $i$ for which $\Delta_{i} \leq M$. By the space bound for $k$-mergers in Theorem 38.2 we see that the space used by link $i$ is dominated by the $\Theta\left(s_{i} k_{i}\right)=\Theta\left(k_{i}{ }^{4}\right)$ space use of $S_{i 1}, \ldots, S_{i k_{i}}$. Since $k_{i+1}=\Theta\left(k_{i}^{4 / 3}\right)$, the space used by link $i$ grows double-exponentially with $i$. Hence, $\Delta_{i}$ is a sum of double-exponentially increasing terms and is therefore dominated by its last term. In other words, $\Delta_{i}=\Theta\left(k_{i}{ }^{4}\right)=\Theta\left(s_{i}{ }^{4 / 3}\right)$. By the definition of $i_{M}$ we have $\Delta_{i_{M}} \leq M<\Delta_{i_{M}+1}$. Using $s_{i+1}=\Theta\left(s_{i}^{4 / 3}\right)$ we see that $\log _{M}\left(s_{i_{M}}\right)=\Theta(1)$.

Now consider an element in an input buffer of $K_{i}$. If $i \leq i_{M}$ the element will not get charged at all in our charging scheme, since no memory transfers are used to fill buffers in the links that fit in fast memory. So assume $i>i_{M}$. In that case the element will get charged for the ascent through $K_{i}$ to $B_{i}$ and then through $v_{j}$ to $A_{j}$ for $j=i, i-1, \ldots, i_{M}$. First consider the cost of ascending through $K_{i}$ : By Theorem 38.2, an invocation of the root of $K_{i}$ to fill $B_{i}$ with $k_{i}^{3}$ elements incurs $O\left(k_{i}+\frac{k_{i}{ }^{3}}{B} \log _{M / B} k_{i}{ }^{3}\right)$ memory transfers altogether. Since $M<\Delta_{i_{M}+1}=\Theta\left(k_{i_{M}+1}^{4}\right)$ we have $M=O\left(k_{i}{ }^{4}\right)$. By the tall cache assumption $M=\Omega\left(B^{2}\right)$ we get $B=O\left(k_{i}{ }^{2}\right)$, which implies $k_{i}=O\left(k_{i}{ }^{3} / B\right)$. Under the assumption that no exhaustions occur, i.e., that buffers are filled completely, it follows that an element is charged $O\left(\frac{1}{B} \log _{M / B} k_{i}{ }^{3}\right)=O\left(\frac{1}{B} \log _{M / B} s_{i}\right)$ memory transfers to ascend through $K_{i}$ and into $B_{i}$. Next consider the cost of ascending through $v_{j}$, that is, insertion into $A_{j}$, for $j=i, i-1, \ldots, i_{M}$ : Filling of $A_{j}$ incurs $O\left(1+\left|A_{j}\right| / B\right)$ memory transfers. Since $B=$ $O\left(k_{i_{M}+1}{ }^{2}\right)=O\left(k_{i_{M}}{ }^{8 / 3}\right)$ and $\left|A_{j}\right|=k_{j}{ }^{3}$, this is $O\left(\left|A_{j}\right| / B\right)$ memory transfers, so an element is charged $O(1 / B)$ memory transfers for each $A_{j}$ (under the assumption of no exhaustions). It only remains to bound the number of such buffers $A_{j}$, i.e., to bound $i-i_{M}$. From $s_{i}^{4 / 3}<s_{i+1}$ we have $s_{i_{M}}^{(4 / 3)^{i-i_{M}}}<s_{i}$. Using $\log _{M}\left(s_{i_{M}}\right)=\Theta(1)$ we get $i-i_{M}=O\left(\log \log _{M} s_{i}\right)$. From $\log \log _{M} s_{i}=O\left(\log _{M} s_{i}\right)$ and the tall cache assumption $M=\Omega\left(B^{2}\right)$ we get $i-$ $i_{M}=O\left(\log _{M} s_{i}\right)=O\left(\log _{M / B} s_{i}\right)$. In total we have proved our claim that, assuming no exhaustions occur, an element in an input buffer of $K_{i}$ is charged $O\left(\frac{1}{B} \log _{M / B} s_{i}\right)$ memory transfers during its ascent to $A_{1}$.

We imagine maintaining the credit invariant that each element in a buffer holds enough credits to be able to pay for the ascent from its current position to $A_{1}$, at the cost analyzed above. In particular, an element needs $O\left(\frac{1}{B} \log _{M / B} s_{i}\right)$ credits when it is inserted in an input buffer of $K_{i}$. The cost of these credits we will attribute to the $\operatorname{SwEEP}(i)$ operation inserting it, effectively making all invocations of mergers be prepaid by $\operatorname{SwEEP}(i)$ operations.

A $\operatorname{SwEEP}(i)$ operation also incurs memory transfers by itself; we now bound these. In the $\operatorname{SweEP}(i)$ operation we first form $\sigma_{1}$ by traversing the path $p$ from $A_{1}$ to $S_{i c_{i}}$. Since the links are laid out sequentially in memory, this traversal at most constitutes a linear scan of the consecutive memory locations containing $A_{1}$ through $K_{i}$. Such a scan takes $O\left(\left(\Delta_{i-1}+\left|A_{i}\right|+\left|B_{i}\right|+\left|K_{i}\right|\right) / B\right)=O\left(k_{i}{ }^{3} / B\right)=O\left(s_{i} / B\right)$ memory transfers. Next we form 
$\sigma_{2}$ using DeLEteMin operations; the cost of which is paid for by the credits placed on the elements. Finally, we merge of $\sigma_{1}$ and $\sigma_{2}$ into $\sigma$, and place some of the elements in buffers on $p$ and some of the elements in $S_{i c_{i}}$. The number of memory transfers needed for this is bounded by the $O\left(s_{i} / B\right)$ memory transfers needed to traverse $p$ and $S_{i c_{i}}$. Hence, the memory transfers incurred by the $\operatorname{SwEEP}(i)$ operation itself is $O\left(s_{i} / B\right)$.

After the $\operatorname{SwEEP}(i)$ operation, the credit invariant must be reestablished. Each of the $O\left(s_{i}\right)$ elements inserted into $S_{i c_{i}}$ must receive $O\left(\frac{1}{B} \log _{M / B} s_{i}\right)$ credits. Additionally, the elements inserted into the part of the path $p$ from $A_{1}$ through $A_{i-1}$ must receive enough credits to cover their ascent to $A_{1}$, since the credits that resided with elements in the same positions before the operations were used when forming $\sigma_{2}$ by DELETEMin operations. This constitute $O\left(\Delta_{i-1}\right)=o\left(s_{i}\right)$ elements which by the analysis above must receive $O\left(\frac{1}{B} \log _{M / B} s_{i}\right)$ credits each. Altogether $O\left(s_{i} / B\right)+O\left(\frac{s_{i}}{B} \log _{M / B} s_{i}\right)=O\left(\frac{s_{i}}{B} \log _{M / B} s_{i}\right)$ memory transfers are attributed to a $\operatorname{SwEeP}(i)$ operation, again under the assumption that no exhaustions occur during invocations.

To actually account for exhaustions, that is, the memory transfers incurred when filling buffers that become exhausted, we note that filling a buffer partly incurs at most the same number of memory transfers as filling it entirely. This number was analyzed above to be $O\left(\left|A_{i}\right| / B\right)$ for $A_{i}$ and $O\left(\frac{\left|B_{i}\right|}{B} \log _{M / B} s_{i}\right)$ for $B_{i}$, when $i>i_{M}$. If $B_{i}$ become exhausted, only a $\operatorname{Sweep}(i)$ can remove that status. If $A_{i}$ become exhausted, only a $\operatorname{Sweep}(j)$ for $j \geq i$ can remove that status. As at most a $\operatorname{single} \operatorname{SwEEP}(j)$ with $j>i$ can take place between one $\operatorname{SwEEP}(i)$ and the next, $B_{i}$ can only become exhausted once for each $\operatorname{SwEEP}(i)$, and $A_{i}$ can only become exhausted twice for each $\operatorname{SwEep}(i)$. From $\left|A_{i}\right|=\left|B_{i}\right|=k_{i}{ }^{3}=\Theta\left(s_{i}\right)$ it follows that charging $\operatorname{SwEEP}(i)$ an additional cost of $O\left(\frac{s_{i}}{B} \log _{M / B} s_{i}\right)$ memory transfers will cover all costs of filling buffers when exhaustion occurs.

Overall we have shown that we can account for all memory transfers if we attribute $O\left(\frac{s_{i}}{B} \log _{M / B} s_{i}\right)$ memory transfers to each $\operatorname{SwEEP}(i)$. By induction on $i$, we can show that at least $s_{i}$ insertions have to take place between each $\operatorname{SwEEP}(i)$. Thus, if we charge the $\operatorname{SwEEP}(i)$ cost to the last $s_{i}$ insertions preceding the $\operatorname{SwEEP}(i)$, each insertion is charged $O\left(\frac{1}{B} \log _{M / B} s_{i}\right)$ memory transfers. Given a sequence of operation on an initial empty priority queue, let $i_{\max }$ be the largest $i$ for which $\operatorname{Sweep}(i)$ takes place. We have $s_{i_{\max }} \leq N$, where $N$ is the number of insertions in the sequence. An insertion can be charged by at most one $\operatorname{SwEeP}(i)$ for $i=1, \ldots, i_{\max }$, so by the double-exponential growth of $s_{i}$, the number of memory transfers charged to an insertion is

$$
O\left(\sum_{k=0}^{\infty} \frac{1}{B} \log _{M / B} N^{(3 / 4)^{k}}\right)=O\left(\frac{1}{B} \log _{M / B} N\right)=O\left(\frac{1}{B} \log _{M / B} \frac{N}{B}\right)
$$

where the last equality follows from the tall cache assumption $M=\Omega\left(B^{2}\right)$.

Finally, we bound the space use of the entire structure. To ensure a space usage linear in $N$, we create a link $i$ when it is first used, i.e., when the first $\operatorname{SwEEP}(i)$ occurs. At that point in time, $c_{i}, A_{i}, v_{i}, B_{i}, K_{i}$, and $S_{i 1}$ are created. These take up $\Theta\left(s_{i}\right)$ space combined. At each subsequent $\operatorname{SwEEP}(i)$ operation, we create the next input buffer $S_{i c_{i}}$ of size $s_{i}$. As noted above, each $\operatorname{SwEEP}(i)$ is preceded by at least $s_{i}$ insertions, from which an $O(N)$ space bound follows. To ensure that the entire structure is laid out in consecutive memory locations, the structure is moved to a larger memory area when it has grown by a constant factor. When allocated, the size of the new memory area is chosen such that it will hold the input buffers $S_{i j}$ that will be created before the next move. The amortized cost of this is $O(1 / B)$ per insertion. 
THEOREM 38.6 Using $\Theta(M)$ fast memory, a sequence of $N$ INSERT, DELETEMIN, and DELETE operations can be performed on an initially empty Funnel Heap using $O(N)$ space in $O\left(\frac{1}{B} \log _{M / B} \frac{N}{B}\right)$ amortized memory transfers each.

Brodal and Fagerberg [16] gave a refined analysis for a variant of the Funnel Heap that shows that the structure adapts to different usage profiles. More precisely, they showed that the $i$ th insertion uses amortized $O\left(\frac{1}{B} \log _{M / B} \frac{N_{i}}{B}\right)$ memory transfers, where $N_{i}$ can be defined in any of the following three ways: $(a) N_{i}$ is the number of elements present in the priority queue when the $i$ th insertion is performed, $(b)$ if the $i$ th inserted element is removed by a DELETEMin operation prior to the $j$ th insertion then $N_{i}=j-i$, or $(c) N_{i}$ is the maximum rank of the $i$ th inserted element during its lifetime in the priority queue, where rank denotes the number of smaller elements in the queue.

\subsubsection{Exponential Level Based Priority Queue}

While the Funnel Heap is inspired by Mergesort and uses $k$-mergers as the basic building block, the exponential level priority queue of Arge et al. [5] is somewhat inspired by distribution sorting and uses sorting as a basic building block.

\section{Structure}

The structure consists of $\Theta(\log \log N)$ levels whose sizes vary from $N$ to some small size $c$ below a constant threshold $c_{t}$; the size of a level corresponds (asymptotically) to the number of elements that can be stored within it. The $i$ 'th level from above has size $N^{(2 / 3)^{i-1}}$ and for convenience we refer to the levels by their size. Thus the levels from largest to smallest are level $N$, level $N^{2 / 3}$, level $N^{4 / 9}, \ldots$, level $X^{9 / 4}$, level $X^{3 / 2}$, level $X$, level $X^{2 / 3}$, level $X^{4 / 9}, \ldots$, level $c^{9 / 4}$, level $c^{3 / 2}$, and level $c$. In general, a level can contain any number of elements less than or equal to its size, except level $N$, which always contains $\Theta(N)$ elements. Intuitively, smaller levels store elements with smaller keys or elements that were more recently inserted. In particular, the minimum key element and the most recently inserted element are always in the smallest (lowest) level $c$. Both insertions and deletions are initially performed on the smallest level and may propagate up through the levels.

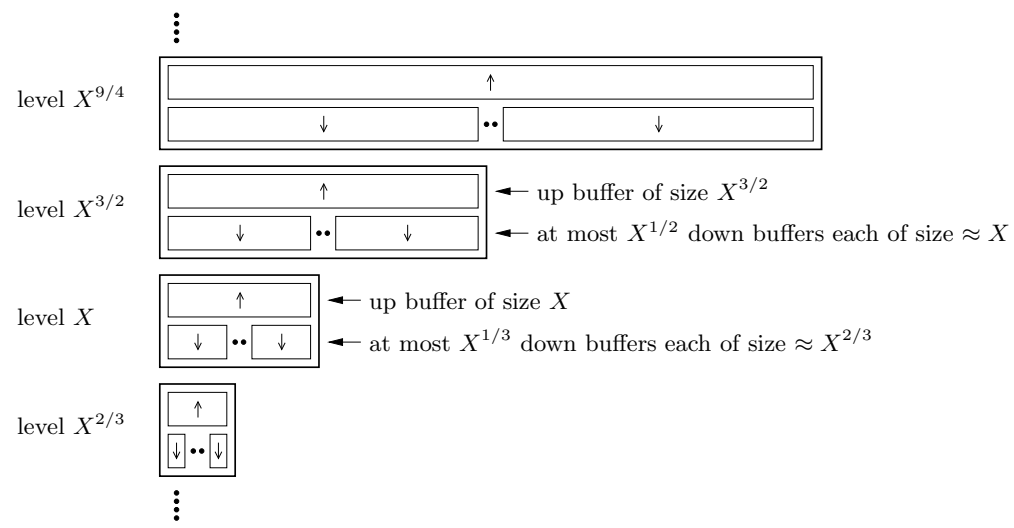

FIGURE 38.11: Levels $X^{2 / 3}, X, X^{3 / 2}$, and $X^{9 / 4}$ of the priority queue data structure. 
Elements are stored in a level in a number of buffers, which are also used to transfer elements between levels. Level $X$ consists of one up buffer $u^{X}$ that can store up to $X$ elements, and at most $X^{1 / 3}$ down buffers $d_{1}^{X}, \ldots, d_{X^{1 / 3}}^{X}$ each containing between $\frac{1}{2} X^{2 / 3}$ and $2 X^{2 / 3}$ elements. Thus level $X$ can store up to $3 X$ elements. We refer to the maximum possible number of elements that can be stored in a buffer as the size of the buffer. Refer to Figure 38.11. Note that the size of a down buffer at one level matches the size (up to a constant factor) of the up buffer one level down.

We maintain three invariants about the relationships between the elements in buffers of various levels:

Invariant 4 At level $X$, elements are sorted among the down buffers, that is, elements in $d_{i}^{X}$ have smaller keys than elements in $d_{i+1}^{X}$, but elements within $d_{i}^{X}$ are unordered.

The element with largest key in each down buffer $d_{i}^{X}$ is called a pivot element. Pivot elements mark the boundaries between the ranges of the keys of elements in down buffers.

Invariant 5 At level $X$, the elements in the down buffers have smaller keys than the elements in the up buffer.

Invariant 6 The elements in the down buffers at level $X$ have smaller keys than the elements in the down buffers at the next higher level $X^{3 / 2}$.

The three invariants ensure that the keys of the elements in the down buffers get larger as we go from smaller to larger levels of the structure. Furthermore, an order exists between the buffers on one level: keys of elements in the up buffer are larger than keys of elements in down buffers. Therefore, down buffers are drawn below up buffers on Figure 38.11. However, the keys of the elements in an up buffer are unordered relative to the keys of the elements in down buffers one level up. Intuitively, up buffers store elements that are "on their way up", that is, they have yet to be resolved as belonging to a particular down buffer in the next (or higher) level. Analogously, down buffers store elements that are "on their way down" - these elements are by the down buffers partitioned into several clusters so that we can quickly find the cluster of smallest key elements of size roughly equal to the next level down. In particular, the element with overall smallest key is in the first down buffer at level $c$.

\section{Layout}

The priority queue is laid out in memory such that the levels are stored consecutively from smallest to largest with each level occupying a single region of memory. For level $X$ we reserve space for exactly $3 X$ elements: $X$ for the up buffer and $2 X^{2 / 3}$ for each possible down buffer. The up buffer is stored first, followed by the down buffers stored in an arbitrary order but linked together to form an ordered linked list. Thus $O\left(\sum_{i=0}^{\log _{3 / 2} \log _{c} N} N^{(2 / 3)^{i}}\right)=O(N)$ is an upper bound on the total memory used by the priority queue.

\section{Operations}

To implement the priority queue operations we use two general operations, push and pull. Push inserts $X$ elements into level $X^{3 / 2}$, and pull removes the $X$ elements with smallest keys from level $X^{3 / 2}$ and returns them in sorted order. An InSERT or a DELETEMin is performed simply by performing a push or pull on the smallest level $c$.

Push. To push $X$ elements into level $X^{3 / 2}$, we first sort the $X$ elements cache-obliviously using $O\left(1+\frac{X}{B} \log _{M / B} \frac{X}{B}\right)$ memory transfers. Next we distribute the elements in the sorted list into the down buffers of level $X^{3 / 2}$ by scanning through the list and simultaneously 
visiting the down buffers in (linked) order. More precisely, we append elements to the end of the current down buffer $d_{i}^{X^{3 / 2}}$, and advance to the next down buffer $d_{i+1}^{X^{3 / 2}}$ as soon as we encounter an element with larger key than the pivot of $d_{i}^{X^{3 / 2}}$. Elements with larger keys than the pivot of the last down buffer are inserted in the up buffer $u^{X^{3 / 2}}$. Scanning through the $X$ elements take $O\left(1+\frac{X}{B}\right)$ memory transfers. Even though we do not scan through every down buffer, we might perform at least one memory transfer for each of the $X^{1 / 2}$ possible buffers. Thus the total cost of distributing the $X$ elements is $O\left(\frac{X}{B}+X^{1 / 2}\right)$ memory transfers.

During the distribution of elements a down buffer may run full, that is, contain $2 X$ elements. In this case, we split the buffer into two down buffers each containing $X$ elements using $O\left(1+\frac{X}{B}\right)$ transfers. We place the new buffer in any free down buffer location for the level and update the linked list accordingly. If the level already has the maximum number $X^{1 / 2}$ of down buffers, we remove the last down buffer $d_{X^{1 / 2}}^{X}$ by inserting its no more than $2 X$ elements into the up buffer using $O\left(1+\frac{X}{B}\right)$ memory transfers. Since $X$ elements must have been inserted since the last time the buffer split, the amortized splitting cost per element is $O\left(\frac{1}{X}+\frac{1}{B}\right)$ transfers. In total, the amortized number of memory transfers used on splitting buffers while distributing the $X$ elements is $O\left(1+\frac{X}{B}\right)$.

If the up buffer runs full during the above process, that is, contains more than $X^{3 / 2}$ elements, we recursively push all of these elements into the next level up. Note that after such a recursive push, $X^{3 / 2}$ elements have to be inserted (pushed) into the up buffer of level $X^{3 / 2}$ before another recursive push is needed.

Overall we can perform a push of $X$ elements from level $X$ into level $X^{3 / 2}$ in $O\left(X^{1 / 2}+\right.$ $\left.\frac{X}{B} \log _{M / B} \frac{X}{B}\right)$ memory transfers amortized, not counting the cost of any recursive push operations; it is easy to see that a push maintains all three invariants.

Pull. To describe how to pull the $X$ smallest keys elements from level $X^{3 / 2}$, we first assume that the down buffers contain at least $\frac{3}{2} X$ elements. In this case the first three down buffers $d_{1}^{X^{3 / 2}}, d_{2}^{X^{3 / 2}}$, and $d_{3}^{X^{3 / 2}}$ contain the between $\frac{3}{2} X$ and $6 X$ smallest elements (Invariants 4 and 5). We find and remove the $X$ smallest elements simply by sorting these elements using $O\left(1+\frac{X}{B} \log _{M / B} \frac{X}{B}\right)$ memory transfers. The remaining between $X / 2$ and $5 X$ elements are left in one, two, or three down buffers containing between $X / 2$ and $2 X$ elements each. These buffers can easily be constructed in $O\left(1+\frac{X}{B}\right)$ transfers. Thus we use $O\left(1+\frac{X}{B} \log _{M / B} \frac{X}{B}\right)$ memory transfers in total. It is easy to see that Invariants $4-6$ are maintained.

In the case where the down buffers contain fewer than $\frac{3}{2} X$ elements, we first pull the $X^{3 / 2}$ elements with smallest keys from the next level up. Because these elements do not necessarily have smaller keys than the, say $U$, elements in the up buffer $u^{X^{3 / 2}}$, we then sort this up buffer and merge the two sorted lists. Then we insert the $U$ elements with largest keys into the up buffer, and distribute the remaining between $X^{3 / 2}$ and $X^{3 / 2}+\frac{3}{2} X$ elements into $X^{1 / 2}$ down buffers containing between $X$ and $X+\frac{3}{2} X^{1 / 2}$ each (such that the $O\left(\frac{1}{X}+\frac{1}{B}\right)$ amortized down buffer split bound is maintained). It is easy to see that this maintains the three invariants. Afterwards, we can find the $X$ minimal key elements as above. Note that after a recursive pull, $X^{3 / 2}$ elements have to be deleted (pulled) from the down buffers of level $X^{3 / 2}$ before another recursive pull is needed. Note also that a pull on level $X^{3 / 2}$ does not affect the number of elements in the up buffer $u^{X^{3 / 2}}$. Since we distribute elements into the down and up buffers after a recursive pull using one sort and one scan of $X^{3 / 2}$ elements, the cost of doing so is dominated by the cost of the recursive pull operation itself. Thus ignoring the cost of recursive pulls, we have shown that a pull of $X$ elements from level $X^{3 / 2}$ down to level $X$ can be performed in $O\left(1+\frac{X}{B} \log _{M / B} \frac{X}{B}\right)$ 
memory transfers amortized, while maintaining Invariants 4-6.

\section{Analysis}

To analyze the amortized cost of an INSERT or DELETEMin operation, we consider the total number of memory transfers used to perform push and pull operations during $\frac{N}{2}$ operations; to ensure that the structure always consists of $O(\log \log N)$ levels and use $O(N)$ space we rebuild it using $O\left(\frac{N}{B} \log _{M / B} \frac{N}{B}\right)$ memory transfers (or $O\left(\frac{1}{B} \log _{M / B} \frac{N}{B}\right)$ transfers per operation) after every $\frac{N}{2}$ operations [5].

The total cost of $\frac{N}{2}$ such operations is analyzed as follows: We charge a push of $X$ elements from level $X$ up to level $X^{3 / 2}$ to level $X$. Since $X$ elements have to be inserted in the up buffer $u^{X}$ of level $X$ between such pushes, and as elements can only be inserted in $u^{X}$ when elements are inserted (pushed) into level $X, O(N / X)$ pushes are charged to level $X$ during the $\frac{N}{2}$ operations. Similarly, we charge a pull of $X$ elements from level $X^{3 / 2}$ down to level $X$ to level $X$. Since between such pulls $\Theta(X)$ elements have to be deleted from the down buffers of level $X$ by pulls on $X, O(N / X)$ pulls are charged to level $X$ during the $\frac{N}{2}$ operations.

Above we argued that a push or pull charged to level $X$ uses $O\left(X^{1 / 2}+\frac{X}{B} \log _{M / B} \frac{X}{B}\right)$ memory transfers. We can reduce this cost to $O\left(\frac{X}{B} \log _{M / B} \frac{X}{B}\right)$ by more carefully examining the costs for differently sized levels. First consider a push or pull of $X \geq B^{2}$ elements into or from level $X^{3 / 2} \geq B^{3}$. In this case $\frac{X}{B} \geq \sqrt{X}$, and we trivially have that $O\left(X^{1 / 2}+\right.$ $\left.\frac{X}{B} \log _{M / B} \frac{X}{B}\right)=O\left(\frac{X}{B} \log _{M / B} \frac{X}{B}\right)$. Next, consider the case $B^{4 / 3} \leq X<B^{2}$, where the $X^{1 / 2}$ term in the push bound can dominate and we have to analyze the cost of a push more carefully. In this case we are working on a level $X^{3 / 2}$ where $B^{2} \leq X^{3 / 2}<B^{3}$; there is only one such level. Recall that the $X^{1 / 2}$ cost was from distributing $X$ sorted elements into the less than $X^{1 / 2}$ down buffers of level $X^{3 / 2}$. More precisely, a block of each buffer may have to be loaded and written back without transferring a full block of elements into the buffer. Assuming $M=\Omega\left(B^{2}\right)$, we from $X^{1 / 2} \leq B$ see that a block for each of the buffers can fit into fast memory. Consequently, if a fraction of the fast memory is used to keep a partially filled block of each buffer of level $X^{3 / 2}\left(B^{2} \leq X^{3 / 2} \leq B^{3}\right)$ in fast memory at all times, and full blocks are written to disk, the $X^{1 / 2}$ cost would be eliminated. In addition, if all of the levels of size less than $B^{2}$ (of total size $O\left(B^{2}\right)$ ) are also kept in fast memory, all transfer costs associated with them would be eliminated. The optimal paging strategy is able to keep the relevant blocks in fast memory at all times and thus eliminates these costs.

Finally, since each of the $O(N / X)$ push and pull operations charged to level $X\left(X>B^{2}\right)$ uses $O\left(\frac{X}{B} \log _{M / B} \frac{X}{B}\right)$ amortized memory transfers, the total amortized transfer cost of an InSERT or DELETEMIN operation in the sequence of $\frac{N}{2}$ such operations is

$$
O\left(\sum_{i=0}^{\infty} \frac{1}{B} \log _{M / B} \frac{N^{(2 / 3)^{i}}}{B}\right)=O\left(\frac{1}{B} \log _{M / B} \frac{N}{B}\right) .
$$

THEOREM 38.7 Using $\Theta(M)$ fast memory, $N$ Insert, DeleteMin, and Delete operations can be performed on an initially empty exponential level priority queue using $O(N)$ space in $O\left(\frac{1}{B} \log _{M / B} \frac{N}{B}\right)$ amortized memory transfers each. 


\subsection{2d Orthogonal Range Searching}

As discussed in Section 38.3, there exist cache-oblivious B-trees that support updates and queries in $O\left(\log _{B} N\right)$ memory transfers (e.g. Theorem 38.5); several cache-oblivious Btree variants can also support (one-dimensional) range queries in $O\left(\log _{B} N+\frac{K}{B}\right)$ memory transfers $[11,12,18]$, but at an increased amortized update cost of $O\left(\log _{B} N+\frac{\log ^{2} N}{B}\right)=$ $O\left(\log _{B}^{2} N\right)$ memory transfers (e.g. Theorem 38.4).

In this section we discuss cache-oblivious data structures for two-dimensional orthogonal range searching, that is, structures for storing a set of $N$ points in the plane such that the points in a axis-parallel query rectangle can be reported efficiently. In Section 38.5.1 we first discuss a cache-oblivious version of a $k d$-tree. This structure uses linear space and answers queries in $O\left(\sqrt{N / B}+\frac{K}{B}\right)$ memory transfers; this is optimal among linear space structures [22]. It supports updates in $O\left(\frac{\log N}{B} \cdot \log _{M / B} N\right)=O\left(\log _{B}^{2} N\right)$ transfers. In Section 38.5.2 we then discuss a cache-oblivious version of a two-dimensional range tree. The structure answers queries in the optimal $O\left(\log _{B} N+\frac{K}{B}\right)$ memory transfers but uses $O\left(N \log ^{2} N\right)$ space. Both structures were first described by Agarwal et al. [1].

\subsubsection{Cache-Oblivious kd-Tree}

\section{Structure}

The cache-oblivious kd-tree is simply a normal kd-tree laid out in memory using the van Emde Boas layout. This structure, proposed by Bentley [13], is a binary tree of height $O(\log N)$ with the $N$ points stored in the leaves of the tree. The internal nodes represent a recursive decomposition of the plane by means of axis-orthogonal lines that partition the set of points into two subsets of equal size. On even levels of the tree the dividing lines are horizontal, and on odd levels they are vertical. In this way a rectangular region $R_{v}$ is naturally associated with each node $v$, and the nodes on any particular level of the tree partition the plane into disjoint regions. In particular, the regions associated with the leaves represent a partition of the plane into rectangular regions containing one point each. Refer to Figure 38.12 .
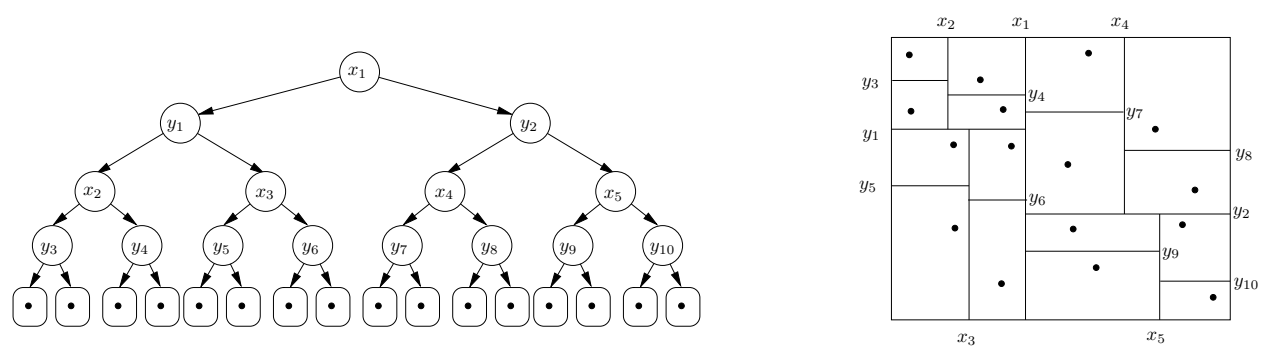

FIGURE 38.12: kd-tree and the corresponding partitioning.

\section{Query}

An orthogonal range query $Q$ on a kd-tree $\mathcal{T}$ is answered recursively starting at the root: At a node $v$ we advance the query to a child $v_{c}$ of $v$ if $Q$ intersects the region $R_{v_{c}}$ associated with $v_{c}$. At a leaf $w$ we return the point in $w$ if it is contained in $Q$. A standard 
argument shows that the number of nodes in $\mathcal{T}$ visited when answering $Q$, or equivalently, the number of nodes $v$ where $R_{v}$ intersects $Q$, is $O(\sqrt{N}+K) ; \sqrt{N}$ nodes $v$ are visited where $R_{v}$ is intersected by the boundary of $Q$ and $K$ nodes $u$ with $R_{u}$ completely contained in $Q[13]$.

If the kd-tree $\mathcal{T}$ is laid out using the van Emde Boas layout, we can bound the number of memory transfers used to answer a query by considering the nodes $\log B$ levels above the leaves of $\mathcal{T}$. There are $O\left(\frac{N}{B}\right)$ such nodes as the subtree $\mathcal{T}_{v}$ rooted in one such node $v$ contains $B$ leaves. By the standard query argument, the number of these nodes visited by a query is $O\left(\sqrt{N / B}+\frac{K}{B}\right)$. Thus, the number of memory transfers used to visit nodes more than $\log B$ levels above the leaves is $O\left(\sqrt{N / B}+\frac{K}{B}\right)$. This is also the overall number of memory transfers used to answer a query, since (as argued in Section 38.2.1) the nodes in $\mathcal{T}_{v}$ are contained in $O(1)$ blocks, i.e. any traversal of (any subset of) the nodes in a subtree $\mathcal{T}_{v}$ can be performed in $O(1)$ memory transfers.

\section{Construction}

In the RAM model, a kd-tree on $N$ points can be constructed recursively in $O(N \log N)$ time; the root dividing line is found using an $O(N)$ time median algorithm, the points are distributed into two sets according to this line in $O(N)$ time, and the two subtrees are constructed recursively. Since median finding and distribution can be performed cacheobliviously in $O(N / B)$ memory transfers [20, 24], a cache-oblivious kd-tree can be constructed in $O\left(\frac{N}{B} \log N\right)$ memory transfers. Agarwal et al. [1] showed how to construct $\log \sqrt{N}=\frac{1}{2} \log N$ levels in $O\left(\operatorname{Sort}_{M, B}(N)\right)$ memory transfers, leading to a recursive construction algorithms using only $O\left(\operatorname{Sort}_{M, B}(N)\right)$ memory transfers.

\section{Updates}

In the RAM model a kd-tree $\mathcal{T}$ can relatively easily be modified to support deletions in $O(\log N)$ time using global rebuilding. To delete a point from $\mathcal{T}$, we simply find the relevant leaf $w$ in $O(\log N)$ time and remove it. We then remove $w$ 's parent and connect $w$ 's grandparent to $w$ 's sibling. The resulting tree is no longer a kd-tree but it still answers queries in $O(\sqrt{N}+T)$ time, since the standard argument still applies. To ensure that $N$ is proportional to the actual number of points in $\mathcal{T}$, the structure is completely rebuilt after $\frac{N}{2}$ deletions. Insertions can be supported in $O\left(\log ^{2} N\right)$ time using the so-called logarithmic method [14], that is, by maintaining $\log N$ kd-trees where the $i$ 'th $\mathrm{kd}$-tree is either empty or of size $2^{i}$ and then rebuilding a carefully chosen set of these structures when performing an insertion.

Deletes in a cache-oblivious kd-tree is basically done as in the RAM version. However, to still be able to load a subtree $\mathcal{T}_{v}$ with $B$ leaves in $O(1)$ memory transfers and obtain the $O\left(\sqrt{N / B}+\frac{K}{B}\right)$ query bound, data locality needs to be carefully maintained. By laying out the kd-tree using (a slightly relaxed version of) the exponential layout (Section 38.3.2) rather than the van Emde Boas layout, and by periodically rebuilding parts of this layout, Agarwal et al. [1] showed how to perform a delete in $O\left(\log _{B} N\right)$ memory transfers amortized while maintaining locality. They also showed how a slightly modified version of the logarithmic method and the $O\left(\operatorname{Sort}_{M, B}(N)\right)$ construction algorithms can be used to perform inserts in $O\left(\frac{\log N}{B} \log _{M / B} N\right)=O\left(\log _{B}^{2} N\right)$ memory transfers amortized.

THEOREM 38.8 There exists a cache-oblivious (kd-tree) data structure for storing a set of $N$ points in the plane using linear space, such that an orthogonal range query can be answered in $O\left(\sqrt{N / B}+\frac{K}{B}\right)$ memory transfers. The structure can be constructed cache- 
obliviously in $O\left(\operatorname{Sort}_{M, B}(N)\right)$ memory transfers and supports updates in $O\left(\frac{\log N}{B} \log _{M / B} N\right)=$ $O\left(\log _{B}^{2} N\right)$ memory transfers.

\subsubsection{Cache-Oblivious Range Tree}

The main part of the cache-oblivious range tree structure for answering (four-sided) orthogonal range queries is a structure for answering three-sided queries $Q=\left[x_{l}, x_{r}\right] \times\left[y_{b}, \infty\right)$, that is, for finding all points with $x$-coordinates in the interval $\left[x_{l}, x_{r}\right]$ and $y$-coordinates above $y_{b}$. Below we discuss the two structures separately.

\section{Three-Sided Queries.}

\section{Structure}

Consider dividing the plane into $\sqrt{N}$ vertical slabs $X_{1}, X_{2}, \ldots, X_{\sqrt{N}}$ containing $\sqrt{N}$ points each. Using these slabs we define $2 \sqrt{N}-1$ buckets. A bucket is a rectangular region of the plane that completely spans one or more consecutive slabs and is unbounded in the positive $y$-direction, like a three-sided query. To define the $2 \sqrt{N}-1$ buckets we start with $\sqrt{N}$ active buckets $b_{1}, b_{2}, \ldots, b_{\sqrt{N}}$ corresponding to the $\sqrt{N}$ slabs. The $x$-range of the slabs define a natural linear ordering on these buckets. We then imagine sweeping a horizontal sweep line from $y=-\infty$ to $y=\infty$. Every time the total number of points above the sweep line in two adjacent active buckets, $b_{i}$ and $b_{j}$, in the linear order falls to $\sqrt{N}$, we mark $b_{i}$ and $b_{j}$ as inactive. Then we construct a new active bucket spanning the slabs spanned by $b_{i}$ and $b_{j}$ with a bottom $y$-boundary equal to the current position of the sweep line. This bucket replaces $b_{i}$ and $b_{j}$ in the linear ordering of active buckets. The total number of buckets defined in this way is $2 \sqrt{N}-1$, since we start with $\sqrt{N}$ buckets and the number of active buckets decreases by one every time a new bucket is constructed. Note that the procedure defines an active $y$-interval for each bucket in a natural way. Buckets overlap but the set of buckets with active $y$-intervals containing a given $y$-value (the buckets active when the sweep line was at that value) are non-overlapping and span all the slabs. This means that the active $y$-intervals of buckets spanning a given slab are non-overlapping. Refer to Figure 38.13(a).

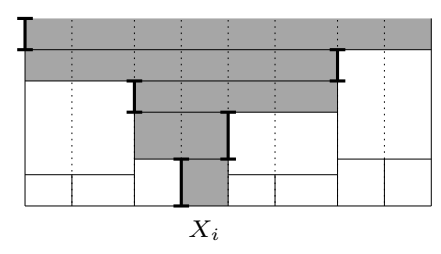

(a)

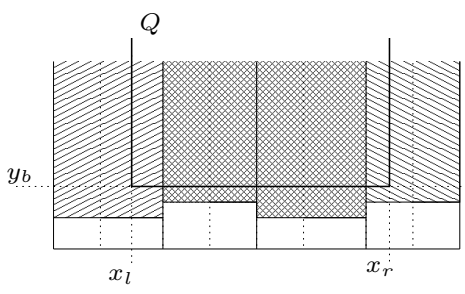

(b)

FIGURE 38.13: (a) Active intervals of buckets spanning slab $X_{i}$; (b) Buckets active at $y_{b}$.

After defining the $2 \sqrt{N}-1$ buckets, we are ready to present the three-sided query data structure; it is defined recursively: It consists of a cache-oblivious B-tree $\mathcal{T}$ on the $\sqrt{N}$ boundaries defining the $\sqrt{N}$ slabs, as well as a cache-oblivious B-tree for each of the $\sqrt{N}$ slabs; the tree $\mathcal{T}_{i}$ for slab $i$ contains the bottom endpoint of the active $y$-intervals of the $O(\sqrt{N})$ buckets spanning the slab. For each bucket $b_{i}$ we also store the $\sqrt{N}$ points in $b_{i}$ in 
a list $\mathcal{B}_{i}$ sorted by $y$-coordinate. Finally, recursive structures $\mathcal{S}_{1}, \mathcal{S}_{2}, \ldots, \mathcal{S}_{2 \sqrt{N}-1}$ are built on the $\sqrt{N}$ points in each of the $2 \sqrt{N}-1$ buckets.

\section{Layout}

The layout of the structure in memory consists of $O(N)$ memory locations containing $\mathcal{T}$, then $\mathcal{T}_{1}, \ldots, \mathcal{T}_{\sqrt{N}}$, and $\mathcal{B}_{1}, \ldots, \mathcal{B}_{2 \sqrt{N}-1}$, followed by the recursive structures $\mathcal{S}_{1}, \ldots, \mathcal{S}_{2 \sqrt{N}-1}$. Thus the total space use of the structure is $S(N) \leq 2 \sqrt{N} \cdot S(\sqrt{N})+O(N)=O(N \log N)$.

\section{Query}

To answer a three-sided query $Q$, we consider the buckets whose active $y$-interval contain $y_{b}$. These buckets are non-overlapping and together they contain all points in $Q$, since they span all slabs and have bottom $y$-boundary below $y_{b}$. We report all points that satisfy $Q$ in each of the buckets with $x$-range completely between $x_{l}$ and $x_{r}$. At most two other buckets $b_{l}$ and $b_{r}$ - the ones containing $x_{l}$ and $x_{r}$ - can contain points in $Q$, and we find these points recursively by advancing the query to $\mathcal{S}_{l}$ and $\mathcal{S}_{r}$. Refer to Figure 38.13(b).

We find the buckets $b_{l}$ and $b_{r}$ that need to be queried recursively and report the points in the completely spanned buckets as follows. We first query $\mathcal{T}$ using $O\left(\log _{B} \sqrt{N}\right)$ memory transfers to find the slab $X_{l}$ containing $x_{l}$. Then we query $\mathcal{T}_{l}$ using another $O\left(\log _{B} \sqrt{N}\right)$ memory transfers to find the bucket $b_{l}$ with active $y$-interval containing $y_{b}$. We can similarly find $b_{r}$ in $O\left(\log _{B} \sqrt{N}\right)$ memory transfers. If $b_{l}$ spans slabs $X_{l}, X_{l+1}, \ldots, X_{m}$ we then query $\mathcal{T}_{m+1}$ with $y_{b}$ in $O\left(\log _{B} \sqrt{N}\right)$ memory transfers to find the active bucket $b_{i}$ to the right of $b_{l}$ completely spanned by $Q$ (if it exists). We report the relevant points in $b_{i}$ by scanning $\mathcal{B}_{i}$ top-down until we encounter a point not contained in $Q$. If $K^{\prime}$ is the number or reported points, a scan of $\mathcal{B}_{i}$ takes $O\left(1+\frac{K^{\prime}}{B}\right)$ memory transfers. We continue this procedure for each of the completely spanned active buckets. By construction, we know that every two adjacent such buckets contain at least $\sqrt{N}$ points above $y_{b}$. First consider the part of the query that takes place on recursive levels of size $N \geq B^{2}$, such that $\sqrt{N} / B \geq \log _{B} \sqrt{N} \geq 1$. In this case the $O\left(\log _{B} \sqrt{N}\right)$ overhead in finding and processing two consecutive completely spanned buckets is smaller than the $O(\sqrt{N} / B)$ memory transfers used to report output points; thus we spend $O\left(\log _{B} \sqrt{N}+\frac{K_{i}}{B}\right)$ memory transfers altogether to answer a query, not counting the recursive queries. Since we perform at most two queries on each level of the recursion (in the active buckets containing $x_{l}$ and $x_{r}$ ), the total cost over all levels of size at least $B^{2}$ is $O\left(\sum_{i=1}^{\log \log _{B} N} \log _{B} N^{1 / 2^{i}}+\frac{K_{i}}{B}\right)=O\left(\log _{B} N+\frac{K}{B}\right)$ transfers. Next consider the case where $N=B$. In this case the whole level, that is, $\mathcal{T}, \mathcal{T}_{1}, \ldots, \mathcal{T}_{\sqrt{B}}$ and $\mathcal{B}_{1}, \ldots, \mathcal{B}_{2 \sqrt{B}-1}$, is stored in $O(B)$ contiguously memory memory locations and can thus be loaded in $O(1)$ memory transfers. Thus the optimal paging strategy can ensure that we only spend $O(1)$ transfers on answering a query. In the case where $N \leq \sqrt{B}$, the level and all levels of recursion below it occupies $O(\sqrt{B} \log \sqrt{B})=O(B)$ space. Thus the optimal paging strategy can load it and all relevant lower levels in $O(1)$ memory transfers. This means that overall we answer a query in $O\left(\log _{B} N+\frac{K}{B}\right)$ memory transfers, provided that $N$ and $B$ are such that we have a level of size $B^{2}$ (and thus of size $B$ and $\sqrt{B}$ ); when answering a query on a level of size between $B$ and $B^{2}$ we cannot charge the $O\left(\log _{B} \sqrt{N}\right)$ cost of visiting two active consecutive buckets to the $(<B)$ points found in the two buckets. Agarwal et al. [1] showed how to guarantee that we have a level of size $B^{2}$ by assuming that $B=2^{2^{d}}$ for some non-negative integer $d$. Using a somewhat different construction, Arge et al. [6] showed how to remove this assumption. 
THEOREM 38.9 There exists a cache-oblivious data structure for storing $N$ points in the plane using $O(N \log N)$ space, such that a three-sided orthogonal range query can be answered in $O\left(\log _{B} N+\frac{K}{B}\right)$ memory transfers.

Four-sided queries.

Using the structure for three-sided queries, we can construct a cache-oblivious range tree structure for four-sided orthogonal range queries in a standard way. The structure consists of a cache-oblivious B-tree $\mathcal{T}$ on the $N$ points sorted by $x$-coordinates. With each internal node $v$ we associate a secondary structure for answering three-sided queries on the points stored in the leaves of the subtree rooted at $v$ : If $v$ is the left child of its parent then we have a three-sided structure for answering queries with the opening to the right, and if $v$ is the right child then we have a three-sided structure for answering queries with the opening to the left. The secondary structures on each level of the tree use $O(N \log N)$ space, for a total space usage of $O\left(N \log ^{2} N\right)$.

To answer an orthogonal range query $Q$, we search down $\mathcal{T}$ using $O\left(\log _{B} N\right)$ memory transfers to find the first node $v$ where the left and right $x$-coordinate of $Q$ are contained in different children of $v$. Then we query the right opening secondary structure of the left child of $v$, and the left opening secondary structure of the right child of $v$, using $O\left(\log _{B} N+\frac{K}{B}\right)$ memory transfers. Refer to Figure 38.14. It is easy to see that this correctly reports all $K$ points in $Q$.

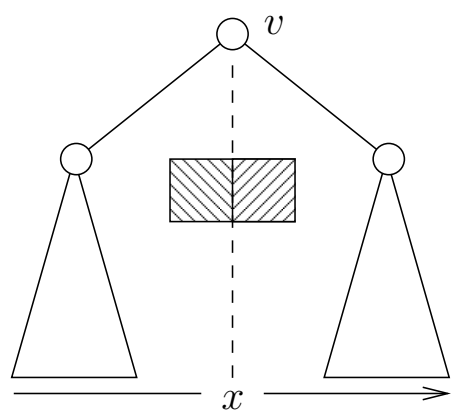

FIGURE 38.14: Answering a four-sided query in $v$ using two three-sided queries in $v$ 's children.

THEOREM 38.10 There exists a cache-oblivious data structure for storing $N$ points in the plane using $O\left(N \log ^{2} N\right)$ space, such that an orthogonal range query can be answered in $O\left(\log _{B} N+\frac{K}{B}\right)$ memory transfers. 


\section{Acknowledgements}

Lars Arge was supported in part by the National Science Foundation through ITR grant EIA-0112849, RI grant EIA-9972879, CAREER grant CCR-9984099, and U.S.-Germany Cooperative Research Program grant INT-0129182.

Gerth Stølting Brodal was supported by the Carlsberg Foundation (contract number ANS-0257/20), BRICS (Basic Research in Computer Science, www.brics.dk, funded by the Danish National Research Foundation), and the Future and Emerging Technologies programme of the EU under contract number IST-1999-14186 (ALCOM-FT).

Rolf Fagerberg was supported by BRICS (Basic Research in Computer Science, www. brics.dk, funded by the Danish National Research Foundation), and the Future and Emerging Technologies programme of the EU under contract number IST-1999-14186 (ALCOM-FT). Part of this work was done while at University of Aarhus.

\section{References}

[1] P. K. Agarwal, L. Arge, A. Danner, and B. Holland-Minkley. Cache-oblivious data structures for orthogonal range searching. In Proc. 19th ACM Symposium on Computational Geometry, pages 237-245. ACM Press, 2003.

[2] A. Aggarwal and J. S. Vitter. The input/output complexity of sorting and related problems. Communications of the ACM, 31(9):1116-1127, Sept. 1988.

[3] A. Andersson and T. W. Lai. Fast updating of well-balanced trees. In Proc. 2nd Scandinavian Workshop on Algorithm Theory, volume 447 of Lecture Notes in Computer Science, pages 111-121. Springer, 1990.

[4] L. Arge. External memory data structures. In J. Abello, P. M. Pardalos, and M. G. C. Resende, editors, Handbook of Massive Data Sets, pages 313-358. Kluwer Academic Publishers, 2002.

[5] L. Arge, M. Bender, E. Demaine, B. Holland-Minkley, and J. I. Munro. Cache-oblivious priority-queue and graph algorithms. In Proc. 34th ACM Symposium on Theory of Computation, pages 268-276. ACM Press, 2002.

[6] L. Arge, G. S. Brodal, and R. Fagerberg. Improved cache-oblivious two-dimensional orthogonal range searching. Unpublished results, 2004.

[7] R. Bayer and E. McCreight. Organization and maintenance of large ordered indexes. Acta Informatica, 1:173-189, 1972.

[8] M. Bender, E. Demaine, and M. Farach-Colton. Efficient tree layout in a multilevel memory hierarchy. In Proc. 10th Annual European Symposium on Algorithms, volume 2461 of Lecture Notes in Computer Science, pages 165-173. Springer, 2002. Full version at http://www.cs.sunysb.edu/ bender/pub/treelayout-full.ps.

[9] M. A. Bender, G. S. Brodal, R. Fagerberg, D. Ge, S. He, H. Hu, J. Iacono, and A. López-Ortiz. The cost of cache-oblivious searching. In Proc. 44th Annual IEEE Symposium on Foundations of Computer Science, pages 271-282. IEEE Computer Society Press, 2003.

[10] M. A. Bender, R. Cole, and R. Raman. Exponential structures for cache-oblivious algorithms. In Proc. 29th International Colloquium on Automata, Languages, and Programming, Lecture Notes in Computer Science, pages 195-207. Springer, 2002.

[11] M. A. Bender, E. D. Demaine, and M. Farach-Colton. Cache-oblivious B-trees. In Proc. 41st Annual IEEE Symposium on Foundations of Computer Science, pages 339-409. IEEE Computer Society Press, 2000.

[12] M. A. Bender, Z. Duan, J. Iacono, and J. Wu. A locality-preserving cache-oblivious 
dynamic dictionary. In Proc. 13th Annual ACM-SIAM Symposium on Discrete Algorithms, pages 29-38. SIAM, 2002.

[13] J. L. Bentley. Multidimensional binary search trees used for associative searching. Communication of the ACM, 18:509-517, 1975.

[14] J. L. Bentley. Decomposable searching problems. Information Processing Letters, 8(5):244-251, 1979.

[15] G. S. Brodal and R. Fagerberg. Cache oblivious distribution sweeping. In Proc. 29th International Colloquium on Automata, Languages, and Programming, Lecture Notes in Computer Science, pages 426-438. Springer, 2002.

[16] G. S. Brodal and R. Fagerberg. Funnel heap - a cache oblivious priority queue. In Proc. 13th International Symposium on Algorithms and Computation, volume 2518 of Lecture Notes in Computer Science, pages 219-228. Springer, 2002.

[17] G. S. Brodal and R. Fagerberg. On the limits of cache-obliviousness. In Proc. 35th ACM Symposium on Theory of Computation, pages 307-315. ACM Press, 2003.

[18] G. S. Brodal, R. Fagerberg, and R. Jacob. Cache oblivious search trees via binary trees of small height. In Proc. 13th Annual ACM-SIAM Symposium on Discrete Algorithms, pages 39-48. SIAM, 2002.

[19] G. S. Brodal, R. Fagerberg, and K. Vinther. Engineering a cache-oblivious sorting algorithm. In Proc. 6th Workshop on Algorithm Engineering and Experiments, 2004.

[20] M. Frigo, C. E. Leiserson, H. Prokop, and S. Ramachandran. Cache-oblivious algorithms. In Proc. 40th Annual IEEE Symposium on Foundations of Computer Science, pages 285-298. IEEE Computer Society Press, 1999.

[21] A. Itai, A. G. Konheim, and M. Rodeh. A sparse table implementation of priority queues. In Proc. 8th International Colloquium on Automata, Languages, and Programming, volume 115 of Lecture Notes in Computer Science, pages 417-431. Springer, 1981.

[22] K. V. R. Kanth and A. K. Singh. Optimal dynamic range searching in non-replicating index structures. In Proc. International Conference on Database Theory, volume 1540 of Lecture Notes in Computer Science, pages 257-276. Springer, 1999.

[23] R. E. Ladner, R. Fortna, and B.-H. Nguyen. A comparison of cache aware and cache oblivious static search trees using program instrumentation. In Experimental Algorithmics, From Algorithm Design to Robust and Efficient Software (Dagstuhl seminar, September 2000), volume 2547 of Lecture Notes in Computer Science, pages 78-92. Springer, 2002.

[24] H. Prokop. Cache-oblivious algorithms. Master's thesis, Massachusetts Institute of Technology, Cambridge, MA, June 1999.

[25] N. Rahman, R. Cole, and R. Raman. Optimized predecessor data structures for internal memory. In Proc. 3rd Workshop on Algorithm Engineering, volume 2141 of Lecture Notes in Computer Science, pages 67-78. Springer, 2001.

[26] P. van Emde Boas. Preserving order in a forest in less than logarithmic time and linear space. Information Processing Letters, 6:80-82, 1977.

[27] J. S. Vitter. External memory algorithms and data structures: Dealing with massive data. ACM Computing Surveys, 33(2):209-271, June 2001. 


\section{Index}

cache oblivious, 38-1-38-27

$k$-merger, 38-5-38-7

$1 \mathrm{~d}$ range queries, 38-4

$2 \mathrm{~d}$ orthogonal range searching, 38-2038- 25

exponential tree layout, $38-10-38-12$

model, 38-2

priority queues, $38-12-38-20$

search trees, 38-7-38-12

density based, 38-8-38-10

exponential tree based, 38-10-3812

searching, 38-3

sorting, 38-7

Funnel Heap, 38-13

Funnelsort, 38-7

search trees

low height, 38-8-38-9

tall cache assumption, 38-2

van Emde Boas layout, 38-3 